\title{
Synthesis and oxidative rearrangement of selenenylated dihydropyrans
}

\author{
Sébastien Redon, ${ }^{\mathrm{a}}$ Xavier Pannecoucke, ${ }^{\mathrm{a}}$ Xavier Franck, ${ }^{* a}$ Francis Outurquin $* a$ \\ DOI: $10.1039 / b 000000 x$
}

Selenenylated dihydropyrans prepared by inverse demand Hetero-Diels-Alder reactions undergo

5 oxidative rearrangement when treated with $\mathrm{H}_{2} \mathrm{O}_{2}$, leading to tetrahydrofuran-2-ones by ring

contraction.

\author{
${ }^{a}$ Universite de Rouen, INSA de Rouen, \\ 10 CNRS UMR 6014, C.O.B.R.A. - I.R.C.O.F \\ 1 Rue Tesniere; 76131 Mont-Saint-Aignan cedex, France. \\ Fax: 33 (0)235522959; Tel: 33 (0)235522402; \\ E-mail:xavier.franck@insa-rouen.fr,francis.outurquin@univ-rouen.fr
}

15

\section{Introduction}

Oxidized organoselenium derivatives are source of molecular diversity by the richness of their chemistry. ${ }^{1}$ Indeed, oxidation of aliphatic organoselenium derivatives is often used to introduce an insaturation by $\beta$-elimination (selenoxide) or to substitute the organoselenium residue by nucleophilic displacement (eg. activation with NBS and substitution by an amine). ${ }^{2}$ Allylic or 20 propargylic phenylselanyl derivatives, when treated by an oxidant, undergo [2,3]-sigmatropic rearrangement leading to allylic alcohols (selenoxide), amines (selenylimine) or halides (halo-adduct). ${ }^{3}$ On the other hand, vinylic or aromatic phenylselanyl derivatives can be oxidized to the selenoxide, which can be isolated in certain cases, or give rise to allenols by [2,3]-sigmatropic rearrangement in the case of 1,3 -dienes. ${ }^{4}$

In the continuation of our studies on the reactivity of selenenylated carbonyl derivatives of type $\mathbf{1},{ }^{4}$ we became interested in 25 their use as dienes in Hetero-Diels-Alder reactions and studied the particular reactivity of these cycloadducts toward oxidants. Maybe owing to the sensibility of selenenylated derivatives to Lewis acids or heat, only few examples of Diels-Alder reactions with $\alpha$-phenylselanyl-enals, -enones or dienes have been described. ${ }^{5}$

\section{Results and discussion}

Inverse Demand Hetero-Diels-Alder reactions have been conducted mainly with electron-rich alkenes (vinyl ethers), leading 30 to 2-alkoxy-5-phenylselanyl-3,4-dihydro-2H-pyrans 3a-l in excellent yields (table 1, entries 1-19).

In these cases, 2-phenylselenylated enals (table 1, entries 1-11) proved to be more reactive than the corresponding enones (table 1, entries 12-19) and the reaction worked well with 3-10 mol\% of the commonly used Eu(FOD) 3 as catalyst, at either room temperature or $50^{\circ} \mathrm{C}$. When $\mathrm{R}^{1}=$ aryl (table 1 , entries $6-11$ ), the reaction is slower and high pressure can help shortening the reaction time. In these cases, no catalyst was necessary. Cyclic vinyl-ether such as dihydrofuran can also be used (table 1 , entries 35 3-5), leading to bicyclic compound 3c-d in good yields. Bulkier vinylethers such as $n$-butyl- and even $t$-butyl- one can be used without loss of efficiency (table 1, entries 9-10). With less reactive phenylselenylated enones (table 1, entries 12-19), the reaction became slower and high pressure was needed to maintain efficiency, especially when $\mathrm{R}^{1}$ or $\mathrm{R}^{2}$ was an aryl group. When both $\mathrm{R}^{1}$ and $\mathrm{R}^{2}$ are aryl groups (table 1, entries 18-19), no reaction occured at all in the absence of high pressure. In order to have access to thio- or phenylselanyl- acetals, ethylvinylsulfide (table 1, entries 20-23) and phenylvinylselenide (table 1, entry 24) were

40 engaged as dienophile in hetero-Diels-Alder reactions. The reactions were always high yielding and high pressure was only needed in the case of enone substituted by a phenyl group (table 1, entry 23); thus 2-ethylsulfanyl-5-phenylselanyl-3,4-dihydro$2 \mathrm{H}$-pyrans 3m-p and the bis-selenenylated 2-ethylselanyl-5-phenylselanyl-3,4-dihydro-2H-pyran 3q could be obtained. With less electron rich dienophiles such as vinylacetate or styrene, Hetero-Diels-Alder reactions with reactive enal never proceeded with vinylacetate, (table 1, entry 25) and required high pressure and longer reaction time for styrene, leading to 2-phenyl-545 phenylselanyl-3,4-dihydro-2H-pyrans 3s (table 1, entries 26-27). It is noteworthy that in this case, no polymerisation of styrene was observed.

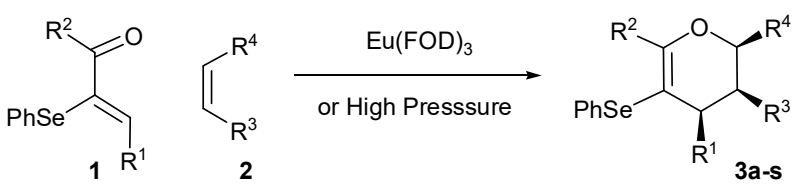

Scheme1 Hetero-Diels-Alder between 2-phenylselanyl-enals or -enones $\mathbf{1}$ and an alkene $\mathbf{2}$ leading to dihydropyrans $\mathbf{3 a - s .}$ 
Once having the cycloadducts in hand, we studied their oxidation. Using standard mild conditions $\left(\mathrm{H}_{2} \mathrm{O}_{2} 35 \%\right.$ in water, 4 eq., in $\left.\mathrm{CH}_{2} \mathrm{Cl}_{2}\right)$ the oxidation of acetals $\left(\mathrm{R}^{4}=\mathrm{OR}\right)$ ended up with an unprecedented ring contraction affording, when $\mathrm{R}^{2}=\mathrm{H}$, 5 -alkoxytetrahydrofuran-2-ones 5 . From acetal $3 \mathbf{e}$ where $\mathrm{R}^{2}=\mathrm{Me}$ and $\mathrm{R}^{4}=\mathrm{OR}$, a mixture of 5-alkoxy-tetrahydro-furan-2-ones $5 \mathbf{e}$ and acetylfuran 6 was observed (Scheme 2).

5 Table 1 Synthesis of dihydropyrans 3

\begin{tabular}{|c|c|c|c|c|c|c|}
\hline entry & $\mathrm{R}^{1}$ & $\mathrm{R}^{2}$ & $\mathrm{R}^{3}$ & $\mathrm{R}^{4}$ & conditions & $\begin{array}{c}\text { Compound (yield } \\
\% \text { ) }\end{array}$ \\
\hline 1 & $\mathrm{Me}$ & $\mathrm{H}$ & $\mathrm{H}$ & OEt & $4 \% \operatorname{Eu}(\mathrm{FOD})_{3}$, r.t., $12 \mathrm{~h}$ & 3a (83) \\
\hline 2 & $\mathrm{CH}_{2} \mathrm{OTBDMS}$ & $\mathrm{H}$ & $\mathrm{H}$ & OEt & $3 \% \mathrm{Eu}(\mathrm{FOD})_{3}$, r.t., $12 \mathrm{~h}$ & $3 \mathbf{b}(86)$ \\
\hline 3 & $\mathrm{Me}$ & $\mathrm{H}$ & \multicolumn{2}{|c|}{$-\mathrm{CH}_{2} \mathrm{CH}_{2} \mathrm{O}-$} & $1.0 \mathrm{Gpa}, 50^{\circ} \mathrm{C}, 36 \mathrm{~h}$ & $3 c(84)$ \\
\hline 4 & $\mathrm{Me}$ & $\mathrm{H}$ & \multicolumn{2}{|c|}{$-\mathrm{CH}_{2} \mathrm{CH}_{2} \mathrm{O}-$} & $5 \% \mathrm{Eu}(\mathrm{FOD})_{3}, 40^{\circ} \mathrm{C}, 12 \mathrm{~h}$ & $3 c(78)$ \\
\hline 5 & $\mathrm{H}$ & $\mathrm{H}$ & \multicolumn{2}{|c|}{$-\mathrm{CH}_{2} \mathrm{CH}_{2} \mathrm{O}-$} & $3 \% \operatorname{Eu}(\mathrm{FOD})_{3}$, r.t., $12 \mathrm{~h}$ & 3d (73) \\
\hline 6 & $\mathrm{Ph}$ & $\mathrm{H}$ & $\mathrm{H}$ & OEt & $10 \% \mathrm{Eu}(\mathrm{FOD})_{3}, 40^{\circ} \mathrm{C}, 48 \mathrm{~h}$ & 3e (90) \\
\hline 7 & $\mathrm{Ph}$ & $\mathrm{H}$ & $\mathrm{H}$ & OEt & $1.0 \mathrm{Gpa}$, r.t., $15 \mathrm{~h}$ & $3 e(50)$ \\
\hline 8 & $\mathrm{Ph}$ & $\mathrm{H}$ & $\mathrm{H}$ & OEt & $1.0 \mathrm{Gpa}, 50^{\circ} \mathrm{C}, 10 \mathrm{~h}$ & 3e (99) \\
\hline 9 & $\mathrm{Ph}$ & $\mathrm{H}$ & $\mathrm{H}$ & $\mathrm{O} n-\mathrm{Bu}$ & $5 \% \mathrm{Eu}(\mathrm{FOD})_{3}, 80^{\circ} \mathrm{C}, 12 \mathrm{~h}$ & $\mathbf{3 f}(98)$ \\
\hline 10 & $\mathrm{Ph}$ & $\mathrm{H}$ & $\mathrm{H}$ & $\mathrm{O} t-\mathrm{Bu}$ & $10 \% \mathrm{Eu}(\mathrm{FOD})_{3}, 80^{\circ} \mathrm{C}, 12 \mathrm{~h}$ & $3 g(97)$ \\
\hline 11 & $p$-OMePh & $\mathrm{H}$ & $\mathrm{H}$ & OEt & $5 \% \mathrm{Eu}(\mathrm{FOD})_{3}, 40^{\circ} \mathrm{C}, 12 \mathrm{~h}$ & $3 h(89)$ \\
\hline 12 & $\mathrm{Me}$ & $\mathrm{Me}$ & $\mathrm{H}$ & $\mathrm{OEt}$ & $10 \% \mathrm{Eu}(\mathrm{FOD})_{3}, 40^{\circ} \mathrm{C}, 48 \mathrm{~h}$ & $3 \mathbf{i}(93)$ \\
\hline 13 & $\mathrm{Me}$ & $\mathrm{Me}$ & $\mathrm{H}$ & OEt & $1.0 \mathrm{Gpa}$, r.t., $20 \mathrm{~h}$ & $3 \mathbf{i}(93)$ \\
\hline 14 & $\mathrm{Me}$ & $\mathrm{Ph}$ & $\mathrm{H}$ & OEt & $10 \% \mathrm{Eu}(\mathrm{FOD})_{3}, 40^{\circ} \mathrm{C}, 20 \mathrm{~h}$ & 3j (35) \\
\hline 15 & $\mathrm{Me}$ & $\mathrm{Ph}$ & $\mathrm{H}$ & OEt & $1.2 \mathrm{Gpa}, 50^{\circ} \mathrm{C}, 20 \mathrm{~h}$ & $3 \mathbf{j}(91)$ \\
\hline 16 & $\mathrm{Ph}$ & $\mathrm{Me}$ & $\mathrm{H}$ & OEt & $10 \% \mathrm{Eu}(\mathrm{FOD})_{3}, 40^{\circ} \mathrm{C}, 48 \mathrm{~h}$ & $3 \mathbf{k}(46)$ \\
\hline 17 & $\mathrm{Ph}$ & $\mathrm{Me}$ & $\mathrm{H}$ & $\mathrm{OEt}$ & $1.2 \mathrm{Gpa}, 50^{\circ} \mathrm{C}, 20 \mathrm{~h}$ & $3 \mathbf{k}(94)$ \\
\hline 18 & $\mathrm{Ph}$ & $\mathrm{Ph}$ & $\mathrm{H}$ & OEt & $10 \% \mathrm{Eu}(\mathrm{FOD})_{3}$, r.t., $48 \mathrm{~h}$ & 31 (N.R.) \\
\hline 19 & $\mathrm{Ph}$ & $\mathrm{Ph}$ & $\mathrm{H}$ & $\mathrm{OEt}$ & $1.2 \mathrm{Gpa}, 50^{\circ} \mathrm{C}, 20 \mathrm{~h}$ & $3 \mathbf{l}(88)$ \\
\hline 20 & $\mathrm{Me}$ & $\mathrm{H}$ & $\mathrm{H}$ & SEt & $4 \% \operatorname{Eu}(\mathrm{FOD})_{3}$, r.t., $12 \mathrm{~h}$ & $3 \mathbf{m}(95)$ \\
\hline 21 & $\mathrm{Ph}$ & $\mathrm{H}$ & $\mathrm{H}$ & SEt & $10 \% \mathrm{Eu}(\mathrm{FOD})_{3}, 40^{\circ} \mathrm{C}, 12 \mathrm{~h}$ & 3n (90) \\
\hline 22 & $\mathrm{Me}$ & $\mathrm{Me}$ & $\mathrm{H}$ & SEt & $10 \% \operatorname{Eu}(\mathrm{FOD})_{3}$, r.t., $12 \mathrm{~h}$ & $30(91)$ \\
\hline 23 & $\mathrm{Ph}$ & $\mathrm{Me}$ & $\mathrm{H}$ & SEt & $1.2 \mathrm{Gpa}, 40^{\circ} \mathrm{C}, 20 \mathrm{~h}$ & $3 \mathbf{p}(90)$ \\
\hline 24 & $\mathrm{Me}$ & $\mathrm{H}$ & $\mathrm{H}$ & $\mathrm{SePh}$ & $10 \% \mathrm{Eu}(\mathrm{FOD})_{3}, 80^{\circ} \mathrm{C}, 24 \mathrm{~h}$ & $3 q(76)$ \\
\hline 25 & $\mathrm{Me}$ & $\mathrm{H}$ & $\mathrm{H}$ & $\mathrm{OCOCH}_{3}$ & $1.0 \mathrm{Gpa}, 50^{\circ} \mathrm{C}, 24 \mathrm{~h}$ & $3 r$ (N.R.) \\
\hline 26 & $\mathrm{Me}$ & $\mathrm{H}$ & $\mathrm{H}$ & $\mathrm{Ph}$ & $1.2 \mathrm{Gpa}, 50^{\circ} \mathrm{C}, 16 \mathrm{~h}$ & 3s (24) \\
\hline 27 & $\mathrm{Me}$ & $\mathrm{H}$ & $\mathrm{H}$ & $\mathrm{Ph}$ & $1.2 \mathrm{Gpa}, 50^{\circ} \mathrm{C}, 48 \mathrm{~h}$ & $3 s(64)$ \\
\hline
\end{tabular}

In order to elucidate the mechanism and characterize intermediates, we performed the oxidation of $3 \mathbf{a}$ in EtOAc or THF and, in these cases, only the selenoxide $\mathbf{4 a}$ could be obtained, without rearrangement. When this selenoxide $\mathbf{4 a}$ was reacted with $\mathrm{H}_{2} \mathrm{O}_{2}$ in $\mathrm{CH}_{2} \mathrm{Cl}_{2}$, rearrangement cleanly occurred, allowing us to propose that $\mathbf{4 a}$ is the first intermediate in the rearrangement. 10 Dihydropyran 3s $\left(\mathrm{R}^{4}=\mathrm{Ph}\right)$ was also submitted to $\mathrm{H}_{2} \mathrm{O}_{2}$ oxidation in $\mathrm{CH}_{2} \mathrm{Cl}_{2}$ and we only isolated acid $7 \mathbf{s}$, resulting from an oxidative cleavage of the double bond. In the case of dihydropyran $3 \mathbf{l}\left(\mathrm{R}^{1}=\mathrm{R}^{2}=\mathrm{Ph}\right)$, only the selenoxide $4 \mathbf{l}$ could be obtained and no rearrangement occurred. When we used simple dihydropyran with no phenylselanyl residue, no oxidation occurred at all and starting material was recovered unchanged (not shown). This surprising oxidation susceptibility of such 5-phenylselanyl-3,4dihydro- $2 H$-pyrans 3 is due to the presence of the selenium residue and is reported for the first time.

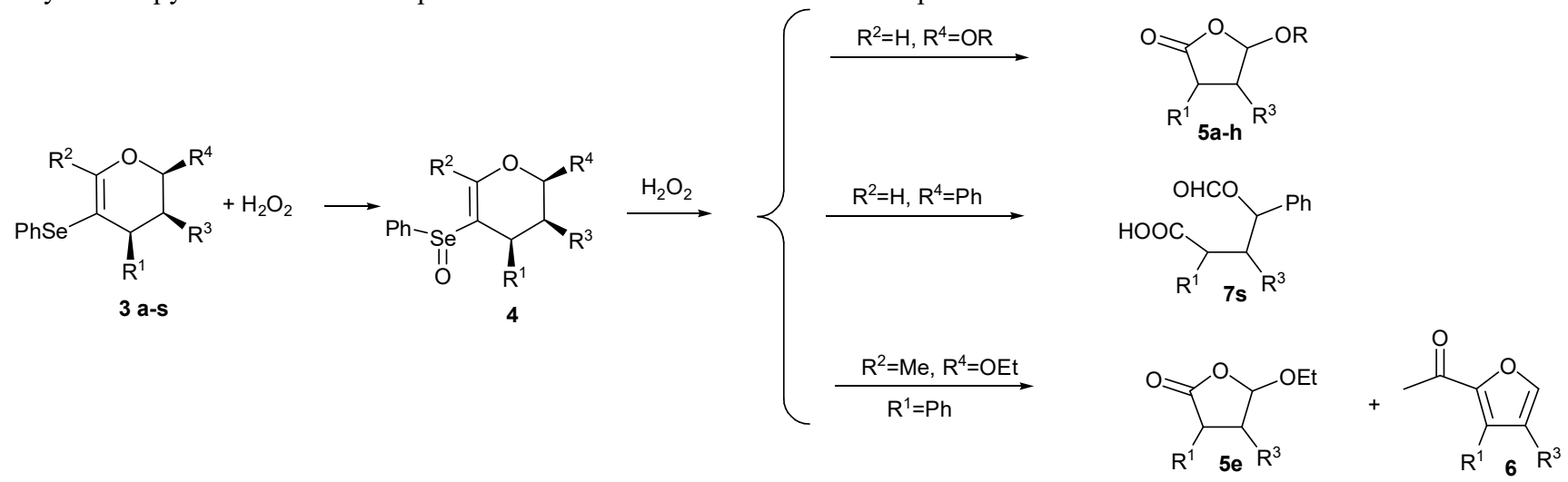

Scheme 2 Different products arising from oxidation of dihydropyrans 3. 
<smiles>[R]C1=C([R5](=O)P)C([R1])C([R])C([R])O1</smiles><smiles>[R]C1OC(C)C([R])C([R])C2([R5](=O)[O-])OC2([R])O1</smiles><smiles>[R]C1C(OCC)OC([R5]([H])=O)(C(C)=O)C1[R]</smiles>

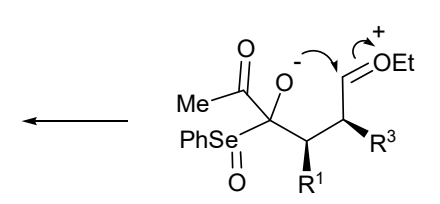<smiles>[R]C1OC(O)C(O)([R5](=O)P)C([R])C1[R]</smiles><smiles>[R]C1OC(O)C(=O)C([R])C1[R]</smiles>

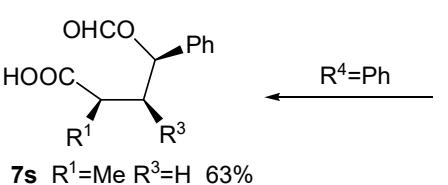<smiles>[R]C1OC(O)OC(=O)C([R])C1[R]</smiles><smiles>[R]OC(I)C(=O)O</smiles><smiles>[R]C(C)C([R])C([R])C(=O)O</smiles>
5 b $^{1}=\mathrm{CH}_{2} \mathrm{OTBDMS} \mathrm{R} \mathrm{R}^{3}=\mathrm{HR} \mathrm{R}=\mathrm{Et} \quad 42 \%$ 5c R $\mathrm{R}^{1}=\mathrm{Me} \quad \mathrm{R}^{3}-\mathrm{R}=\left(\mathrm{CH}_{2}\right)_{2} \quad 36 \%$ 5d $\mathrm{R}^{1}=\mathrm{H} \quad \mathrm{R}^{3}-\mathrm{R}=\left(\mathrm{CH}_{2}\right)_{2} \quad 30 \%$ 5e $\mathrm{R}^{1}=\mathrm{Ph} \quad \mathrm{R}^{3}=\mathrm{H} \mathrm{R}=\mathrm{Et} \quad 44 \%$ 5f $\mathrm{R}^{1}=\mathrm{Ph} \quad \mathrm{R}^{3}=\mathrm{H} \mathrm{R}=n-\mathrm{Bu} 24 \%$ $5 \mathrm{~g} \mathrm{R}^{1}=\mathrm{Ph} \quad \mathrm{R}^{3}=\mathrm{H} \mathrm{R}=t-\mathrm{Bu} \quad 30 \%$ $5 \mathrm{R} \mathrm{R}^{1}=p-\mathrm{MeO}-\mathrm{C}_{6} \mathrm{H}_{4} \quad \mathrm{R}^{3}=\mathrm{HR}$ R=Et $\quad 43 \%$

Scheme 3 Proposed mechanism.

Having identified intermediates and characterized different reaction products according to the substitution of the dihydropyrans $\mathbf{3}$, 5 we can now propose a mechanism.

The presence of the selenium residue, first oxidized to the corresponding selenoxide 4 , is then responsible for the high polarisation of the enolic double bond, which can certainly be epoxidized by $\mathrm{H}_{2} \mathrm{O}_{2}$. Then, depending on the substitution on position $6\left(\mathrm{R}^{2}=\mathrm{H}\right.$ or $\left.\mathrm{R}^{2}=\mathrm{Me}\right)$, two different pathways can be observed (Scheme 3$)$.

First, when $\mathrm{R}^{2}=\mathrm{H}$, water (solvent of $\mathrm{H}_{2} \mathrm{O}_{2}$ ) can attack at the position 6 to give, after expulsion of "PhSeOH", the keto10 hemiacetal 8. $\mathrm{PhSeOH}$ can then be further oxidized by the excess of $\mathrm{H}_{2} \mathrm{O}_{2}$ to $\mathrm{PhSe}(\mathrm{O}) \mathrm{OOH}$, phenylperseleninic acid, which is know as a strong but selective oxidant able to perform Baeyer-Villiger reactions. ${ }^{6}$ Indeed, Baeyer-Villiger oxidation of the ketohemiacetal 8 gives rise to the correponding lactone-hemiacetal $\mathbf{9}^{7}$ which can rearrange with expulsion of formic acid and ring closure of the carboxylate onto the formed oxonium. This mechanism is supported by the isolation of carboxylic acid $\mathbf{7 s}$, from the reaction of dihydropyran $3 \mathrm{~s}$ where $\mathrm{R}^{4}=\mathrm{Ph}$. Indeed, the same lactone-hemiacetal can be drawn but, in this case, $\mathrm{R}^{4}$ is not electron

15 donating enough to open the ring and fragmentation occurs, leading to carboxylic acid $\mathbf{7} \mathbf{S}$ with an hydroxyl group functionalized as a formyl ester.

Second, when $\mathrm{R}^{2}=\mathrm{Me}$, the attack of water on the epoxide is slowed down (but still exists) by steric factors, thus the second pathway occurs thanks to the electron donating abitily of $\mathrm{R}^{4}=\mathrm{OEt}$ which can help opening the 6-membered cycle and form an oxonium ion. The liberated hydroxyl in position 5 can then attack the oxonium, giving rise to a five-membered ring (5-exo-trig). 20 This rearrangement is similar to the one observed by Armstrong et al. in the synthesis of tetrahydrofuranones. ${ }^{8}$ Then the selenoxide can $\beta$-eliminate to give a dihydro-furan which can further lose $\mathrm{EtOH}$ to yield the corresponding furan 6.

Both patways are competitive and depend on the substitution pattern $\left(\mathrm{R}^{2}\right)$. The presence of the selenium residue increases the reactivity of these dihydropyrans and makes possible the whole oxidation process to occur in very mild conditions. 5-alkoxytetrahydrofuran-2-ones 5 have been prepared in moderate yields, what can be explained by the relative instability of the

25 intermediates and by the presence of water which can trap oxonium ions. All attemps to remove water out of the reaction were unsuccessfull (use of urea- $\mathrm{H}_{2} \mathrm{O}_{2}$ complex).

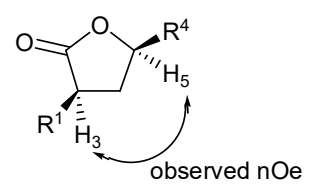

5e cis minor<smiles>[R1][C@H]1C[C@]([Z1])(C)OC1=O</smiles>

5e trans major

$\mathrm{R}^{1}=\mathrm{Ph}, \mathrm{R}^{4}=\mathrm{OEt}$ 
Scheme 4: Stereochemistry of tetrahydrofuran-2-one $\mathbf{5 e .}$

Concerning the diastereoselectivity of the rearrangement through the oxocarbenium ion, it appears that the resulting tetrahydrofuran-2-ones $\mathbf{5 a - b}$ and $\mathbf{5 e - h}$ are isolated as a main trans diastereomer ( $>90 / 10$ in all cases), as the minor cis shows nOe 5 correlations between H-3 and H-5 (scheme 4). On the contrary, the stereochemistry of tetrahydrofuran-2-ones 5c-d is all cis, the spectrosopic data being in accordance with previously published data. ${ }^{9}$

\section{Conclusion}

To conclude, we have developped an efficient route toward selenenylated dihydropyrans through a high yielding inverse demand Hetero-Diels-Alder. This pathway allowed a general access to various dihydropyrans including thio- and seleno-acetals. ${ }_{10}$ Further oxidation of these selenenylated dihydropyrans $\left(\mathrm{R}^{4}=\mathrm{OR}\right)$ with $\mathrm{H}_{2} \mathrm{O}_{2}$ proceeded with unprecedented rearrangement, affording 5-alkoxy-tetrahydro-furan-2-ones 5 through a contraction of the intermediate selenoxide 4 . A mechanism was postulated, passing through an epoxide which could then be transformed either in furan moiety or in 5-alkoxy-tetrahydrofuran-2one, depending on the substitution pattern.

\section{Experimental}

\section{${ }_{15}$ General methods}

NMR spectra were recorded on a Bruker DPX 300 spectrometer operating at $300 \mathrm{MHz}$ for proton and $75.4 \mathrm{MHz}$ for carbon. This probe is equipped with pulsedfield $(z)$ gradients. ${ }^{77} \mathrm{Se}$ NMR spectra were recorded at $21{ }^{\circ} \mathrm{C}$ on a Bruker DPX 400 spectrometer operating at $76.29 \mathrm{MHz}$ for ${ }^{77} \mathrm{Se}$, using a pulse length of $19 \mu \mathrm{s}\left(90^{\circ}\right.$ pulse $\left.=19 \mu \mathrm{s}\right)$ and an optimized relaxation delay of $2 \mathrm{~s}$. An average of 1500 scans for ${ }^{77} \mathrm{Se}$ NMR was necessary to have reliable information. Chemical shifts $(\delta)$ are expressed in ${ }_{20} \mathrm{ppm}$ relative to TMS for ${ }^{1} \mathrm{H}$ and ${ }^{13} \mathrm{C}$ nuclei and to $\mathrm{Me}_{2} \mathrm{Se}$ for ${ }^{77} \mathrm{Se}$ nuclei; coupling constants $(J)$ are given in Hertz; coupling multiplicities are reported using conventional abbreviations. Elemental analyses were obtained on a Carlo-Erba 1106 analysor and Mass Spectra on a HP5890 (electronic impact 70eV) using GC-MS coupling with a Jeol AX 500.

\section{Typical procedure for the synthesis of dihydropyrans 3.} 25

\section{Method under High Pressure.}

The enal 1a $(1 \mathrm{mmol}, 225 \mathrm{mg})$ in ethyl vinyl ether $(10 \mathrm{mmol}, 0.96 \mathrm{ml})$ was introduced in a high-pressure vessel and compressed at the desired pressure and temperature. After decompression, the mixture was concentrated under vacuum. The residue was chromatographied on silicagel (eluent: Ethyl acetate /Cyclohexane: 3/97) to give $282 \mathrm{mg}$ (95\%) of 2-Ethoxy-4-methyl-5-phenylselanyl-3,4-dihydro-2H30 pyran 3a.

\section{Method with Eu(FOD)3.}

To a mixture of enal 1a $(1 \mathrm{mmol}, 225 \mathrm{mg})$ in ethyl vinyl ether $(10 \mathrm{mmol}, 0.96 \mathrm{ml})$, Eu(FOD) $3(2 \%, 21 \mathrm{mg})$ was introduced under nitrogen and the mixture stirred at the desired temperature. After the reaction was completed, controlled by TLC, the mixture was concentrated 35 under vacuum. The residue was chromatographied on silicagel (eluent: Ethyl Acetate/Cyclohexane: 3/97) to give $275 \mathrm{mg}$ (93\%) of 2Ethoxy-4-methyl-5-phenylselanyl-3,4-dihydro-2H-pyran 3a.

2-Ethoxy-4-methyl-5-phenylselanyl-3,4-dihydro-2H-pyran (3a). yield=93\%; yellow oil; $R_{f}=0.24$ (AcOEt/Cyclohexane: $\left.5 / 95\right)$; ${ }^{77} \mathrm{Se}$ NMR $\delta(\mathrm{ppm}) 331.7 ;{ }^{1} \mathrm{H}$ NMR $\delta(\mathrm{ppm}) 1.14$ (d, $\left.J=7.0 \mathrm{~Hz}, 3 \mathrm{H}\right), 1.25$ (t, $\left.J=7.0 \mathrm{~Hz}, 3 \mathrm{H}\right), 1.82$ (ddd, 1H, $\left.J=6.2,6.4,13.6 \mathrm{~Hz}, \mathrm{H}-3\right), 2.13$ $40(\mathrm{ddd}, 1 \mathrm{H}, J=2.5,6.9,15.1 \mathrm{~Hz}, \mathrm{H}-3), 2.40(\mathrm{ddd}, 1 \mathrm{H}, J=1.5,6.9,13.6 \mathrm{~Hz}, \mathrm{H}-4), 3.58(\mathrm{~m}, 1 \mathrm{H}), 3.91(\mathrm{~m}, 1 \mathrm{H}), 5.10(\mathrm{dd}, J=2.5,6.2 \mathrm{~Hz}, 1 \mathrm{H}, \mathrm{H}-$ 2), $6.82(\mathrm{~d}, J=1.7 \mathrm{~Hz}, 1 \mathrm{H}, \mathrm{H}-6), 7.17-7.25(\mathrm{~m}, 3 \mathrm{H}), 7.40-7.44(\mathrm{~m}, 2 \mathrm{H}) .{ }^{13} \mathrm{C}$ NMR $(\delta \mathrm{ppm}) 15.4,20.6,30.3(\mathrm{C}-4), 36.3(\mathrm{C}-3), 64.6,98.8$ (C-2), 108.9 (C-5), 126.2, 129.1, 129.8, 132.6, 148.3 (C-6). IR (neat): 2975, 2928, 2873, 1622, 1578, 1476, 1438, 1373, 1130, 1070, 990, 840, 736, $691 \mathrm{~cm}^{-1}$; MS (EI, $\left.70 \mathrm{eV}\right) \mathrm{m} / z$ : 298 (48), 296 (25), 226 (100), 224 (53), 197 (15), 157 (50, PhSe $\left.{ }^{+}\right), 117$ (46), 115 (26), 91 (17), 78 (32), 77 (38, $\left.\mathrm{Ph}^{+}\right), 51$ (41), 41 (45). Anal. Calcd for $\mathrm{C}_{14} \mathrm{H}_{18} \mathrm{O}_{2} \mathrm{Se}$ (297.25): C, 56.57; H, 6.10. Found: C, 56.63; $\mathrm{H}, 6.07$.

${ }_{45}$ 2-Ethoxy-3,4-dihydro-4-[(tert-butyl)dimethylsilyl-methoxy]-5-phenylselanyl-2H-pyran (3b). yield $=86 \%$; yellow oil; $R_{f}=0.41$ (AcOEt/cyclohexane: $3 / 97) ;{ }^{77} \mathrm{Se} \mathrm{NMR} \delta(\mathrm{ppm}) 339.2 \mathrm{ppm} ;{ }^{1} \mathrm{H}$ NMR $\delta(\mathrm{ppm})-0.04$ (s, 3H), $-0.02(\mathrm{~s}, 3 \mathrm{H}), 0.83$ (s, $\left.9 \mathrm{H}\right), 1.24(\mathrm{t}, J=7.0 \mathrm{~Hz}$, $3 \mathrm{H}), 1.97-2.05(\mathrm{~m}, 1 \mathrm{H}, \mathrm{H}-3), 2.16-2.25(\mathrm{~m}, 1 \mathrm{H}, \mathrm{H}-3), 2.43(\mathrm{~m}, 1 \mathrm{H}, \mathrm{H}-4), 3.52-3.67(\mathrm{~m}, 2 \mathrm{H}), 3.81-3.91(\mathrm{~m}, 2 \mathrm{H}), 5.12(\mathrm{dd}, J=2.70,4.1 \mathrm{~Hz}$, $1 \mathrm{H}, \mathrm{H}-2), 6.86(\mathrm{~d}, J=1.65 \mathrm{~Hz}, 1 \mathrm{H}, \mathrm{H}-6), 7.19-7.26(\mathrm{~m}, 3 \mathrm{H}), 7.38-7.40(\mathrm{~m}, 2 \mathrm{H}) .{ }^{13} \mathrm{C}$ NMR $(\delta \mathrm{ppm})-5.2,15.4,18.4,26.1,30.1(\mathrm{C}-3), 37.7$ (C-4), 64.5, 64.9, 98.4 (C-2), 103.7 (C-5), 126.3, 129.1, 129.8, 132.4, 149.8 (C-6); IR (neat): 2954, 2920, 2856, 1621, 1578, 1476, 1438, ${ }_{50}$ 1378, 1360, 1253, 1227, 1130, 1066, 1022, 995, 838, 776, 734, $690 \mathrm{~cm}^{-1}$; MS (EI, $\left.70 \mathrm{eV}\right) \mathrm{m} / z: 428\left(\mathrm{M}^{+}, 6\right), 371\left(\mathrm{M}^{+}-\mathrm{C}(\mathrm{Me}) 3,4\right), 297$ (M+OSiTBDM, 10), 237 (20), 219 (14), 157 ( $\mathrm{PhSe}^{+}$, 22), 73 (100); Anal. Calcd for $\mathrm{C}_{20} \mathrm{H}_{32} \mathrm{O}_{3} \mathrm{SeSi}$ (427.50): C, 56.19; H, 7.54. Found: C, $56.43 ; \mathrm{H}, 7.45$.

\section{4-Methyl-5-phenylselanyl-2,3,3a,7a-tetrahydro-4H-furo}

[2,3-b]pyran (3c). yield=84\%; yellow oil; $R_{f}=0.28$ (AcOEt/Cyclohexane: 5/95); ${ }^{1} \mathrm{H}$ NMR $\delta(\mathrm{ppm}) 1.06(\mathrm{~d}, J=7.1 \mathrm{~Hz}, 3 \mathrm{H}), 1.80-1.98(\mathrm{~m}$, $\left.{ }_{55} 2 \mathrm{H}\right), 2.49(\mathrm{~m}, 1 \mathrm{H}, \mathrm{H}-3), 2.87(\mathrm{~m}, 1 \mathrm{H}, \mathrm{H}-4), 3.98(\mathrm{~m}, 1 \mathrm{H}), 4.21(\mathrm{~m}, 1 \mathrm{H}), 5.49$ (d, J=3.7 Hz, 1H, H-2), 6.84 (d, $\left.J=2.0 \mathrm{~Hz}, 1 \mathrm{H}, \mathrm{H}-6\right), 7.17-$ $7.25(\mathrm{~m}, 3 \mathrm{H}), 7.41-7.45(\mathrm{~m}, 2 \mathrm{H}) .{ }^{13} \mathrm{C}$ NMR $(\delta \mathrm{ppm})$ 18.4, 23.6, $30.2(\mathrm{C}-4), 44.8(\mathrm{C}-3), 68.4,100.5(\mathrm{C}-2), 104.9(\mathrm{C}-5), 126.3,129.0$, 130.0, 132.3, 148.6 (C-6). IR (neat): 2955, 2974, 2360, 1622, 1615, 1575, 1476, 1465, 1436, 1373, 1336, 1308, 1170, 1099, 1043, 1022, 995, 922, 891, 836, 736, $691 \mathrm{~cm}^{-1}$; MS (EI, $\left.70 \mathrm{eV}\right) \mathrm{m} / \mathrm{z}: 296$ (38), 294 (20), 226 (100), 224 (51), $157\left(\mathrm{PhSe}^{+}, 33\right), 117$ (47), 77 (44, $\left.\mathrm{Ph}^{+}\right)$, 51 (47), 41(80). Anal. Calcd for $\mathrm{C}_{14} \mathrm{H}_{16} \mathrm{O}_{2} \mathrm{Se}$ (295.23): C, 56.95; H, 5.46. Found: C, 56.72; H, 5.40 .

${ }_{60}$ 5-Phenylselanyl-2.3,3a,7a-tetrahydro-4H-furo[2,3-b]pyran $\quad$ (3d). yield $=73 \%$; white cristal; m.p. $=48-50^{\circ} \mathrm{C} ; \quad R_{f}=0.29$ (AcOEt/Cyclohexane: 5/95); ${ }^{1} \mathrm{H}$ NMR $\delta$ (ppm) 1.84-2.05 (m, 2H, H-3), 2.27 (d, 1H, J=17.7 Hz, H-4), 2.49 (m, 1H, H-9), 2.68 (dd, 1H, $J=1.9,17.7 \mathrm{~Hz}, \mathrm{H}-4), 3.95-4.01$ (m, 1H, H-2), 4.18-4.25 (m, 1H, H-2), 5.40 (d, J=3.8 Hz, 1H, H-8), 6.82 (s, 1H, H-6), 7.18-7.30 (m, 3H), 7.35-7.50 (m, 2H). ${ }^{13} \mathrm{C}$ NMR $(\delta \mathrm{ppm})$ 27.7, 28.3, 38.5 (C-9), 68.5 (C-2), 98.5, 98.7 (C-8), 126.6, 129.2, 130.4, 131.1, 147.5 (C-6). IR 
(neat): $2897,1628,1577,1476,1437,1157,1102,1066,1022,933,918,857,737,691 \mathrm{~cm}^{-1}$; MS (EI, $\left.70 \mathrm{eV}\right) \mathrm{m} / z: 312(32), 310$ (17), 266 (8), 218 (85), 216 (44), 191 (50), 157 (51, PhSe+), 129 (100), 115 (85), 105 (42), 77 (51, $\left.\mathrm{Ph}^{+}\right), 53$ (58), 51 (81), 44 (42). Anal. Calcd for $\mathrm{C}_{13} \mathrm{H}_{14} \mathrm{O}_{2} \mathrm{Se}$ (281.20): C, 55.52; H, 5.02. Found: C, 55.14; H, 5.06.

2-Ethoxy-4-phenyl-5-phenylselanyl-3,4-dihydro-2H-pyran $\quad(3 \mathbf{e}) . \quad$ yield $=99 \% ; \quad$ yellow $\quad$ solid; $\quad$ m.p. $=73-74{ }^{\circ} \mathrm{C} ; \quad R_{f}=0.26$ (AcOEt/cyclohexane: 5/95); ${ }^{77} \mathrm{Se}$ NMR $\delta(\mathrm{ppm}) 348.5 ;{ }^{1} \mathrm{H}$ NMR $\delta(\mathrm{ppm}) 1.21$ (t, $\left.J=7.0 \mathrm{~Hz}, 3 \mathrm{H}\right), 2.19$ (ddd, 1H, $J=2.2,8.1,13.6 \mathrm{~Hz}, \mathrm{H}-$ 3), 2.38 (ddd, $1 \mathrm{H}, J=2.2,6.9,13.6 \mathrm{~Hz}, \mathrm{H}-3), 3.54-3.66(\mathrm{~m}, 2 \mathrm{H}), 3.95(\mathrm{~m}, 1 \mathrm{H}), 5.17(\mathrm{dd}, J=7.4,1.9 \mathrm{~Hz}, 1 \mathrm{H}, \mathrm{H}-2), 7.03(\mathrm{~d}, J=1.7 \mathrm{~Hz}, 1 \mathrm{H}$, $\mathrm{H}-6), 7.06-7.11(\mathrm{~m}, 2 \mathrm{H}), 7.17-7.25(\mathrm{~m}, 6 \mathrm{H}), 7.31-7.35(\mathrm{~m}, 2 \mathrm{H}) .{ }^{13} \mathrm{C}$ NMR $(\delta \mathrm{ppm})$ 15.2, $38.0(\mathrm{C}-3), 42.6(\mathrm{C}-4), 64.6,99.2(\mathrm{C}-2), 106.96$ (C-5), 126.5, 126.7, 128.2, 128.4, 129.0, 130.7, 131.9, 142.8, 149.8 (C-6). IR (KBr): 2982, 2932, 2867, 1603, 1576, 1476, 1437, 1378, 1127, 1040, 906, 768, 730, $700 \mathrm{~cm}^{-1}$; MS (EI, $70 \mathrm{eV)} \mathrm{m/z:} 360$ (15), 358 (8), 314 (10), 288 (33), 286 (19), 237 (14), 179 (36), 178 (36), 10158 (48), $157\left(100, \mathrm{PhSe}^{+}\right), 131$ (78), 128 (56), 115 (44), 103 (31), 78 (64), 77 (73, $\mathrm{Ph}^{+}$), 51 (69), 44 (40). Anal. Calcd for $\mathrm{C}_{19} \mathrm{H}_{20} \mathrm{O}_{2} \mathrm{Se}$ (359.31): C, 63.51; H, 5.61. Found: C, 63.48; H, 5.53.

2-Butoxy-4-phenyl-5-phenylselanyl-3,4-dihydro-2H-pyran (3f). yield=98\%; yellow oil; $\mathrm{R}_{\mathrm{f}}=0.35$ (cyclohexane/EtOAc: $97 / 3$ ), ${ }^{77} \mathrm{Se}$ NMR $\delta(\mathrm{ppm}) 350.0 ;{ }^{1} \mathrm{H}$ NMR $\delta(\mathrm{ppm}) 1.03(\mathrm{t}, J=7.3 \mathrm{~Hz}, 3 \mathrm{H}), 1.47(\mathrm{~m}, 2 \mathrm{H}), 1.68(\mathrm{~m}, 2 \mathrm{H}), 2.33(\mathrm{ddd}, 1 \mathrm{H}, J=1.8,7.9,13.7 \mathrm{~Hz}, \mathrm{H}-3), 2.50$ (ddd, $1 \mathrm{H}, J=2.2,6.9,13.7 \mathrm{~Hz}, \mathrm{H}-3), 3.64(\mathrm{~m}, 1 \mathrm{H}), 3.75(\mathrm{~m}, 1 \mathrm{H}, \mathrm{H}-4), 4.02(\mathrm{~m}, 1 \mathrm{H}), 5.28(\mathrm{dd}, J=2.2,7.3 \mathrm{~Hz}, 1 \mathrm{H}, \mathrm{H}-2), 7.17(\mathrm{~d}, J=1.7 \mathrm{~Hz}$, 15 1H, H-6), 7.20-7.24 (m, 2H), 7.30-7.35 (m, 6H), 7.44-7.50 (m, 2H). ${ }^{13} \mathrm{C}$ NMR ( $\left.\delta \mathrm{ppm}\right) 13.9,19.3,31.7,37.9(\mathrm{C}-3), 42.5(\mathrm{C}-4), 69.0,99.3$ (C-2), 106.8 (C-5), 126.4, 126.6, 128.1, 128.4, 129.0, 130.7, 131.9, 142.9, 149.9 (C-6). IR (neat): 3060, 2959, 2932, 2872, 1622, 1578, 1493, 1476, 1455, 1438, 1372, 1345, 1231, 1123, 1072, 1050, 1021, 911, 839, 823, 736, $699 \mathrm{~cm}^{-1}$; MS (EI, $\left.70 \mathrm{eV}\right) \mathrm{m} / z: 388$ (20), 386 (10), 288 (80), $286(42), 179$ (28), $157\left(\mathrm{PhSe}^{+}, 42\right), 131(92), 115(43), 77\left(38, \mathrm{Ph}^{+}\right), 57$ (34), 41(100). Anal. Calcd for $\mathrm{C}_{21} \mathrm{H}_{24} \mathrm{O}_{2} \mathrm{Se}$ (387.36): C, 65.11; H, 6.24. Found: C, 64.99; H, 6.29.

20 2-tert-Butoxy-4-phenyl-5-phenylselanyl-3,4-dihydro-2H-pyran (3g). yield=97\%; yellow oil; $\mathrm{R}_{\mathrm{f}}=0.35$ (cyclohexane/EtOAc: $97 / 3$ ); ${ }^{77} \mathrm{Se}$ NMR $\delta(\mathrm{ppm}) 347.3 ;{ }^{1} \mathrm{H}$ NMR $\delta(\mathrm{ppm}) 1.34(\mathrm{~s}, 9 \mathrm{H}), 2.28(\mathrm{~m}, 2 \mathrm{H}, \mathrm{H}-3), 3.73(\mathrm{~m}, 1 \mathrm{H}, \mathrm{H}-4), 5.43(\mathrm{dd}, J=2.6,7.2 \mathrm{~Hz}, 1 \mathrm{H}, \mathrm{H}-2), 7.08(\mathrm{~d}$, $J=1.8 \mathrm{~Hz}, 1 \mathrm{H}, \mathrm{H}-6), 7.14-7.17$ (m, 2H), 7.25-7.32 (m, 6H), 7.35-7.40 (m, 2H); ${ }^{13} \mathrm{C}$ NMR $(\delta \mathrm{ppm}) 28.7,39.5(\mathrm{C}-3), 43.2(\mathrm{C}-4), 75.8,94.1$ (C-2), 106.5 (C-5), 126.4, 126.6, 128.1, 128.4, 128.9, 129.3, 132.1, 143.0, 150.3(C-6). IR (neat): 3045, 2958, 2831, 1622, 1614, 1580, $1494,1477,1455,1436,1393,1367,1267,1240,1179,1118,1057,1021,962,916,840,735,699 \mathrm{~cm}^{-1}$; MS (EI, $\left.70 \mathrm{eV}\right) \mathrm{m} / z: 388(7)$,

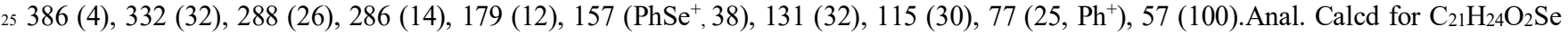
(387.36): C, 65.11; H, 6.24. Found: C, 65.21; H, 6.16.

2-Ethoxy-4-(4-methoxyphenyl)-5-phenylselanyl-3,4-dihydro-2 $\boldsymbol{H}$-pyran (3h). yield=89\%; yellow oil; $R_{f}=0.26$ (AcOEt/cyclohexane: 3/97); ${ }^{1} \mathrm{H}$ NMR $\delta(\mathrm{ppm}) 1.22$ (t, $\left.J=7 ., 0 \mathrm{~Hz}, 3 \mathrm{H}\right), 2.14$ (ddd, 1H, $J=2.2,8.1,13.7 \mathrm{~Hz}, \mathrm{H}-3$ ), 2.33 (ddd, 1H, $J=2.2,6.9,13.7 \mathrm{~Hz}, \mathrm{H}-3$ ), 3.53$3.65(\mathrm{~m}, 2 \mathrm{H}), 3.77(\mathrm{~s}, 3 \mathrm{H}), 3.93(\mathrm{~m}, 1 \mathrm{H}), 5.13(\mathrm{dd}, J=2.2,7.3 \mathrm{~Hz}, 1 \mathrm{H}, \mathrm{H}-2), 6.73-6.76(\mathrm{~m}, 2 \mathrm{H}), 6.97-7.01(\mathrm{~m}, 2 \mathrm{H}), 7.00(\mathrm{~d}, J=1.8 \mathrm{~Hz}, 1 \mathrm{H}$, $\left.{ }_{30} \mathrm{H}-6\right), 7.17-7.21(\mathrm{~m}, 3 \mathrm{H}), 7.31-7.35(\mathrm{~m}, 2 \mathrm{H}) .{ }^{13} \mathrm{C}$ NMR $(\delta \mathrm{ppm})$ 15.2, 38.1 (C-3), $41.9(\mathrm{C}-4), 55.2,64.6,99.2$ (C-2), $107.6(\mathrm{C}-5), 113.5$, 126.4, 129.0, 129.4, 130.7, 131.9, 134.8, 149.5 (C-6), 158.3; IR (neat): 3056, 2975, 2930, 2834, 1614, 1579, 1514, 1476, 1455, 1439, 1378, 1360, 1344, 1303, 1281, 1251, 1177, 1122, 1057, 1033, 913, 876, 830, 780, 736, $691 \mathrm{~cm}^{-1}$; MS (EI, $\left.70 \mathrm{eV}\right) \mathrm{m} / \mathrm{z}: 390(30), 388$ (16), 318 (100), 316 (52), 210 (57), 161 (80), 121 (63), 77 (50, $\mathrm{Ph}^{+}$). Anal. Calcd for $\mathrm{C}_{20} \mathrm{H}_{22} \mathrm{O}_{3} \mathrm{Se}$ (389.34): C, 61.69; H, 5.69. Found: C, 61.55; H, 5.88 .

${ }_{35}$ 2-Ethoxy-3,4-dihydro-4,6-dimethyl-5-phenylselanyl-2H-pyran (3i). yield=93\%; yellow oil; $R_{f}=0.30$ (AcOEt/Cyclohexane: $5 / 95$ ); ${ }^{77} \mathrm{Se}$ NMR $\delta(\mathrm{ppm}) 318.9 ;{ }^{1} \mathrm{H}$ NMR $\delta(\mathrm{ppm}) 1.18(\mathrm{~d}, J=7,1 \mathrm{~Hz}, 3 \mathrm{H}), 1.26(\mathrm{t}, J=6.8 \mathrm{~Hz}, 3 \mathrm{H}), 1.80$ (ddd, $\left.1 \mathrm{H}, J=6.0,6.4,13.6 \mathrm{~Hz}, \mathrm{H}-3\right), 2.11$ (ddd, $1 \mathrm{H}, J=2.3,6.8,13.6 \mathrm{~Hz}, \mathrm{H}-3), 2.12$ (s, 3H), 2.41 (ddd, 1H, $J=1.2,6.8,13.9 \mathrm{~Hz}, \mathrm{H}-4), 3.59$ (m, 1H), $3.92(\mathrm{~m}, 1 \mathrm{H}), 5.08(\mathrm{dd}, J=2.3$, $6.0 \mathrm{~Hz}, 1 \mathrm{H}, \mathrm{H}-2), 7.13-7.25(\mathrm{~m}, 3 \mathrm{H}), 7.30-7.34(\mathrm{~m}, 2 \mathrm{H}) .{ }^{13} \mathrm{C} \mathrm{NMR}(\delta \mathrm{ppm})$ 15.4, 20.7, 21.5, $31.9(\mathrm{C}-4), 36.4(\mathrm{C}-3), 64.4,98.7$ (C-2), 104.1 (C-5), 125.6, 128.7, 129.1, 133.2, 154.9 (C-6). IR (neat): 2975, 2928, 2873, 1638, 1623, 1578, 1476, 1438, 1377, 1236, 1201, 40 1157, 1123, 1096, 1054, 1019, 957, 840, 734, $690 \mathrm{~cm}^{-1}$; MS (EI, $\left.70 \mathrm{eV}\right) \mathrm{m} / \mathrm{z}: 312$ (10), 310 (10), 251 (9), 240 (42), 238 (24), 197 (27), 171 (15), 157 (24, $\left.\mathrm{PhSe}^{+}\right), 116(28), 115$ (33), 91 (21), 77 (38, $\left.\mathrm{Ph}^{+}\right), 51$ (39), 44 (54), 43 (100). Anal. Calcd for $\mathrm{C}_{15} \mathrm{H}_{20} \mathrm{O}_{2} \mathrm{Se}_{(311.28): ~}$, 57.87; H, 6.48. Found: C, 57.53; H, 6.37 .

2-Ethoxy-3,4-dihydro-4-methyl-6-phenyl-5-phenylselanyl-2 $\mathrm{H}$-pyran $\quad \mathbf{( 3 j ) .} \quad$ yield $=91 \% ; \quad$ white $\quad$ solid; $\quad$ m.p. $=66^{\circ} \mathrm{C} ; \quad R_{f}=0.31$ (AcOEt/Cyclohexane: 5/95); ${ }^{77} \mathrm{Se} \mathrm{NMR} \delta$ (ppm) 339.2; ${ }^{1} \mathrm{H}$ NMR $\delta(\mathrm{ppm}) 1.27$ (dd, $\left.J=1.2,6.9 \mathrm{~Hz}, 3 \mathrm{H}\right), 1.29$ (t, $\left.J=7.0 \mathrm{~Hz}, 3 \mathrm{H}\right), 1.93$ (ddd, $\left.{ }_{45} 1 \mathrm{H}, J=5.9,7.0,13.7 \mathrm{~Hz}, \mathrm{H}-3\right), 2.21$ (ddd, $\left.1 \mathrm{H}, J=2.6,7.2,13.7 \mathrm{~Hz}, \mathrm{H}-3\right), 2.49(\mathrm{~m}, 1 \mathrm{H}), 3.67(\mathrm{~m}, 1 \mathrm{H}), 4.03(\mathrm{~m}, 1 \mathrm{H}), 5.24(\mathrm{dd}, J=2.6,5.9$ $\mathrm{Hz}, 1 \mathrm{H}, \mathrm{H}-2), 7.15-7.45(\mathrm{~m}, 10 \mathrm{H}) .{ }^{13} \mathrm{C}$ NMR $(\delta \mathrm{ppm})$ 15.4, 21.7, $31.4(\mathrm{C}-4), 36.3(\mathrm{C}-3), 64.55,99.4(\mathrm{C}-2), 106.7$ (C-5), $126.0,127.7$, 128.8, 129.1, 129.3, 129.9, 132.8, 137.2, 155.2 (C-6). IR (KBr): 3055, 2960, 2926, 1620, 1596, 1578, 1476, 1438, 1372, 1264, 1134, 1093, 1063, 1023, 1008, 996, 884, 831, 765, 736, $696 \mathrm{~cm}^{-1}$; MS (EI, $\left.70 \mathrm{eV}\right) \mathrm{m} / \mathrm{z}: 374$ (14), 302 (40), 300 (20), 221 (10), $157\left(28, \mathrm{PhSe}{ }^{+}\right.$), 145 (44), 105 (100), 77 (58, $\mathrm{Ph}^{+}$), 51 (18). Calcd for $\mathrm{C}_{20} \mathrm{H}_{22} \mathrm{O}_{2} \mathrm{Se}$ (373.34): C, 64.34; H, 5.94. Found: C, 64.49; H, 5.97.

so 2-Ethoxy-3,4-dihydro-6-methyl-4-phenyl-5-phenylselanyl-2H-pyran $\mathbf{( 3 k )}$. yield=94\%; yellow oil; $R_{f}=0.33$ (AcOEt/cyclohexane: $5 / 95) ;{ }^{77} \mathrm{Se}$ NMR $\delta(\mathrm{ppm}) 333.7 ;{ }^{1} \mathrm{H}$ NMR $\delta(\mathrm{ppm}) 1.25(\mathrm{t}, J=7,2 \mathrm{~Hz}, 3 \mathrm{H}), 2.14$ (ddd, $\left.1 \mathrm{H}, J=7.8,9.0,13.6 \mathrm{~Hz}, \mathrm{H}-3\right), 2.24(\mathrm{~d}, J=1.8 \mathrm{~Hz}$, $3 \mathrm{H}), 2.37$ (ddd, 1H, $J=2.2,7.2,13.6 \mathrm{~Hz}, \mathrm{H}-3), 3.57-3.73(\mathrm{~m}, 2 \mathrm{H}), 3.98(\mathrm{~m}, 1 \mathrm{H}), 5.14(\mathrm{dd}, J=2.2,7.8 \mathrm{~Hz}, 1 \mathrm{H}, \mathrm{H}-2), 7.10-7.35(\mathrm{~m}, 10 \mathrm{H})$. ${ }^{13} \mathrm{C}$ NMR $(\delta$ ppm) 15.3, 20.8, 38.9 (C-3), 44.7 (C-4), 64.6, 99.3 (C-2), 102.1 (C-5), 125.9, 126.6, 128.3 (2C overlap), 129.1, 129.4, 132.8, 144.4, 156.8 (C-6). IR (neat): 3059, 2975, 2928, 1694, 1627, 1578, 1476, 1438, 1377, 1233, 1156, 1052, 1022, 871, 761, 691 cm ${ }^{-1}$; MS 55 (EI, $70 \mathrm{eV}$ ) m/z: 374 (8), 372 (4), 302 (24), 300 (13), 259 (11), 179 (22), 178 (26), 157 (14, PhSe ), 145 (30), 128 (18), 115 (16), 77 (23, $\mathrm{Ph}^{+}$), 51 (20), 44 (46), 43 (100). Anal. Calcd for $\mathrm{C}_{20} \mathrm{H}_{22} \mathrm{O}_{2} \mathrm{Se}$ (373.34): C, 64.34; H, 5.94. Found: C, 64.05; H, 5.68.

2-Ethoxy-3,4-dihydro-4,6-diphenyl-5-phenylselanyl-2H-pyran (3l). yield=93\%; yellow oil; $R_{f}=0.40$ (AcOEt/cyclohexane: $\left.5 / 95\right)$; ${ }^{77} \mathrm{Se}$ NMR $\delta(\mathrm{ppm}) 356.0 ;{ }^{1} \mathrm{H}$ NMR $\delta(\mathrm{ppm}) 1.25(\mathrm{t}, J=7.2 \mathrm{~Hz}, 3 \mathrm{H}), 2.20$ (ddd, $1 \mathrm{H}, J=8.2,9.2,17.3 \mathrm{~Hz}, \mathrm{H}-3$ ), 2.42 (ddd, 1H, $J=2.0,7.4,13.6$ $\mathrm{Hz}, \mathrm{H}-3), 3.69(\mathrm{~m}, 1 \mathrm{H}), 4.07(\mathrm{~m}, 1 \mathrm{H}), 5.27$ (dd, $J=2.0,8.2 \mathrm{~Hz}, 1 \mathrm{H}, \mathrm{H}-2), 7.10-7.35(\mathrm{~m}, 13 \mathrm{H}), 7.50-7.53(\mathrm{~m}, 2 \mathrm{H}) .{ }^{13} \mathrm{C} \mathrm{NMR}(\delta \mathrm{ppm}) 15.3$, 6039.1 (C-3), 44.4 (C-4), 64.7, 100.3 (C-2), 105.1 (C-5), 126.5, 126.7, 127.7, 128.4, 128.5, 129.0, 129.1, 129.6, 131.3, 131.9, 136.6, 144.3, 156.8 (C-6). IR (neat) 2973, 2929, 2873, 1691, 1660, 1445, 1376, 1099, 770, 740, $697 \mathrm{~cm}^{-1}$. MS (ESI, positive mode): $\mathrm{m} / \mathrm{z} 451$ and 453 $[\mathrm{M}+\mathrm{H}+\mathrm{O}]^{+}$. Anal. Calcd for $\mathrm{C}_{25} \mathrm{H}_{24} \mathrm{O}_{2} \mathrm{Se}(435.40): \mathrm{C}, 68.96$; H, 5.56. Found: C, 68.76; H, 5.64.

2-Ethylsulfanyl-4-methyl-5-phenylselanyl-3,4-dihydro-2 $\boldsymbol{H}$-pyran (3m). yield=95\%; yellow oil; $R_{f}=0.25$ (EtOAc/Cyclohexane: $5 / 95$ ); ${ }^{1} \mathrm{H}$ NMR $\delta(\mathrm{ppm}): 1.11$ (d, $\left.J=6.9 \mathrm{~Hz}, 3 \mathrm{H}\right), 1.33$ (t, $\left.J=7.6 \mathrm{~Hz}, 3 \mathrm{H}\right), 1.84$ (ddd, $\left.J=4.6,9.4,13.7 \mathrm{~Hz}, 1 \mathrm{H}, \mathrm{H}-3\right), 2.33$ (ddd, $J=2.6,6.3,13.7$ ${ }_{65} \mathrm{~Hz}, 1 \mathrm{H}, \mathrm{H}-3$ '), 2.49 (m, 1H, H-4), 2.77 (m, 2H), 5.24 (dd, $\left.J=2.6,9.4 \mathrm{~Hz}, \mathrm{H}-2\right), 6.90$ (d, J=1.9 Hz, 1H, H-6), 7.18-7.26 (m, 3H), 7.42-7.47 $(\mathrm{m}, 2 \mathrm{H}) .{ }^{13} \mathrm{C}$ NMR $\delta$ (ppm): 15.2, 20.8, 25.1, 31.8 (C-4), 37.65 (C-3), 80.75 (C-2), 108.9 (C-5), 126.3, 129.1, 130.0, 132.3, 150.1 (C-6). IR (neat) : 2963, 2925, 2869, 1607, 1578, 1476, 1438, 1375, 1299, 1266, 1202, 1143, 1112, 1000, 844, 735, $690 \mathrm{~cm}^{-1}$. MS (ESI, positive mode): $\mathrm{m} / \mathrm{z} 431\left([\mathrm{M}+\mathrm{H}+\mathrm{O}]^{+},(63), 429\right.$ (40), 243 (28), $193(30), 191$ (100), 166 (46), 143 (37), 122 (53). Anal. Calcd for $\mathrm{C}_{14} \mathrm{H}_{18} \mathrm{OSSe}$ (313.31): C, 53.66; H, 5.79; S, 10.23. Found: C, 53.34; H, 5.68; S; 10.36. 
2-Ethylsulfanyl-4-phenyl-5-phenylselanyl-3,4-dihydro-2H-pyran (3n). yield=90\%; yellow oil; $R_{f}=0.29$ (EtOAc/Cyclohexane: $5 / 95$ ); ${ }^{1} \mathrm{H}$ NMR $\delta(\mathrm{ppm}): 1.33$ (t, 3H, $\left.J=7.6 \mathrm{~Hz}\right), 2.17-2.29(\mathrm{~m}, 1 \mathrm{H}, \mathrm{H}-3), 2.50$ (ddd, 1H, $\left.J=2.2,6.8,11.7 \mathrm{~Hz}, \mathrm{H}-3\right), 2.70-2.85$ (m, $\left.2 \mathrm{H}\right), 3.67$ (ddd, $J=1.5,6.5,10.2 \mathrm{~Hz}, 1 \mathrm{H}, \mathrm{H}-4), 5.26$ (dd, $1 \mathrm{H}, J=1.9,10.6 \mathrm{~Hz}, \mathrm{H}-2), 7.07-7.10(\mathrm{~m}, 2 \mathrm{H}), 7.09$ (d, $J=1.5 \mathrm{~Hz}, 1 \mathrm{H}, \mathrm{H}-6), 7.18-7.37$ (m, 8H). ${ }^{13} \mathrm{C}$ NMR $\delta$ (ppm): 15.2, 24.8, 39.2 (C-3), 44.4 (C-4), 80.85 (C-2), 107.5 (C-5), 126.6, 127.0, 128.2, 128.4, 129.0, 130.9, 131.8, 142.5 (C-9), 151.6 (C-6). IR (neat): 3060, 2965, 2926, 1607, 1475, 1452, 1437, 1134, 1053, 1021, 758, $737,700 \mathrm{~cm}^{-1}$. MS (ESI): 393 $(\mathrm{M}+\mathrm{H}+\mathrm{O})$.

2-Ethylsulfanyl-4,6-dimethyl-5-phenylselanyl-3,4-dihydro-2 $\boldsymbol{H}$-pyran (3o). yield=91\%; yellow oil; $R_{f}=0.27$ (EtOAc/Cyclohexane: 5/95); ${ }^{1} \mathrm{H}$ NMR $\delta(\mathrm{ppm}): 1.15$ (d, 3H, $\left.J=6.9 \mathrm{~Hz}\right), 1.34(\mathrm{t}, 3 \mathrm{H}, J=7.4 \mathrm{~Hz}), 1.84$ (ddd, $\left.J=4.2,9.4,13.6 \mathrm{~Hz}, 1 \mathrm{H}, \mathrm{H}-3\right), 2.15(\mathrm{~d}, 3 \mathrm{H}, J=1.8$ Hz), 2.30 (ddd, $J=2.4,6.5,13.6 \mathrm{~Hz}, 1 \mathrm{H}, \mathrm{H}-3$ '), 2.50 (m, 1H, H-4), 2.78 (m, 2H), 5.21 (dd, 1H, $J=2.4,9.4 \mathrm{~Hz}, \mathrm{H}-2), 7.15-7.25$ (m, 3H), 10 7.35-7.38 (m, 2H). ${ }^{13} \mathrm{C}$ NMR $\delta$ (ppm): 15.3, 21,0, 21.8, 25.2, 33.3 (C-4), 38.1 (C-3), 80.5 (C-2), 104.4 (C-5), 125.7, 128.9, 129.2, 133.0, 157.2 (C-6). IR (neat): 2970, 2927, 2870, 1610, 1578, 1476, 1438, 1236, 1201, 1151, 1115, 1019, 957, 920, $734,690 \mathrm{~cm}^{-1}$; MS (EI, 70 eV) $m / z$ : 330 (2), 328 (5), 240 (53), 238 (30), 197 (24), 171 (13), 157 (25, $\left.\mathrm{PhSe}^{+}\right), 116$ (28), 115 (34), 88 (30), 77 (24, $\left.\mathrm{Ph}^{+}\right), 60$ (56), 51 (24), 44 (41), 43 (100). Anal. Calcd for $\mathrm{C}_{15} \mathrm{H}_{20} \mathrm{OSSe}$ (327.34): C, 55.03; H, 6.16; S, 9.80. Found: C, 55.05; H, 5.81; $\mathrm{S} 10.13$

2-Ethylsulfanyl-6-methyl-4-phenyl-5-phenylselanyl-3,4-dihydro- $\mathbf{H}$-pyran $\quad \mathbf{( 3 p )}$. yield=88\%; yellow oil; $R_{f}=0.33$ 15 (EtOAc/Cyclohexane: 5/95); ${ }^{1} \mathrm{H}$ NMR $\delta$ (ppm): 1.34 (t, 3H, $\left.J=7.4 \mathrm{~Hz}\right), 2.21$ (d, $\left.J=1.8 \mathrm{~Hz}, 3 \mathrm{H}\right), 2.15-2.25$ (m, $\left.1 \mathrm{H}, \mathrm{H}-3\right), 2.46$ (ddd, $J=1.9$, 6.9, $15.6 \mathrm{~Hz}, 1 \mathrm{H}, \mathrm{H}-3$ ') $2.80(\mathrm{~m}, 2 \mathrm{H}), 3.73(\mathrm{~m}, 1 \mathrm{H}, \mathrm{H}-4), 5.23$ (dd, $1 \mathrm{H}, J=1.9,10.8 \mathrm{~Hz}, \mathrm{H}-2), 7.07-7.10(\mathrm{~m}, 2 \mathrm{H}), 7.18-7.28(\mathrm{~m}, 8 \mathrm{H}) .{ }^{13} \mathrm{C}$ NMR $\delta$ (ppm): 15.3, 20.95, 24.8, 40.2 (C-3), 46.35 (C-4), 80.7 (C-2), 102.8 (C-5), 126.0, 126.85, 128.0, 128.5, 129.1, 129.6, 132.6, 144.1, 158.85 (C-6). IR (neat): 3058, 3026, 2964, 2925, 2869, 1626, 1577, 1492, 1475, 1453, 1436, 1375, 1265, 1211, 1052, 1014, 972, 735, $701 \mathrm{~cm}^{-1}$; MS (ESI, positive mode): m/z 407 ([M+H+O ] $\left.]^{+}, 100\right)$, 405 (55), 319 (29); Anal. Calcd for $\mathrm{C}_{20} \mathrm{H}_{22} \mathrm{OSSe}$ (389.40): C, 61.68; ${ }_{20} \mathrm{H}, 5.69 ; \mathrm{S}, 8.23$. Found: C, 61.35; H, 5.85; S 8.57

4-Methyl-2,5-bis-phenylselanyl-3,4-dihydro-2 $\mathrm{H}$-pyran (3q). yield=80\%; yellow oil; $R_{f}=0.60$ (EtOAc/Cyclohexane: $\left.5 / 95\right) ;{ }^{1} \mathrm{H}$ NMR $\delta$ (ppm): 1.15 (d, 3H, $J=6.9 \mathrm{~Hz}), 1.98-2.07(\mathrm{~m}, 1 \mathrm{H}, \mathrm{H}-3), 2.45-2.59(\mathrm{~m}, 2 \mathrm{H}, \mathrm{H}-4, \mathrm{H}-3), 5.76(\mathrm{dd}, 1 \mathrm{H}, J=3.2,8.2 \mathrm{~Hz}, \mathrm{H}-2), 6.89$ (d, $1 \mathrm{H}$, $J=1.5 \mathrm{~Hz}, \mathrm{H}-6), 7.15-7.67$ (m, 10H). ${ }^{13} \mathrm{C}$ NMR $\delta$ (ppm): 21.1, 31.6 (C-4), 38.2 (C-3), 78.9 (C-2), 109.1 (C-5), 126.4, 128.2, 129.1, 129.2, 129.3, 130.1, 134.5, 135.3, 149.5 (C-6). IR (neat): 3056, 2961, 2922, 1686, 1579, 1476, 1436, 1301, 1229, 1190, 1140, 1107, 1022, 999, $25847,741,690 \mathrm{~cm}^{-1}$.

4-Methyl-2-phenyl-5-phenylselanyl-3,4-dihydro-2 $\boldsymbol{H}$-pyran (3s). yield=64\%; yellow oil; $R_{f}=0.38$ (AcOEt/Cyclohexane: $\left.3 / 97\right)$; ${ }^{1} \mathrm{H}$ NMR $\delta(\mathrm{ppm}) 0.97(\mathrm{~d}, J=7.0 \mathrm{~Hz}, 3 \mathrm{H}), 1.69$ (ddd, $J=2.4,11.5,13.7 \mathrm{~Hz} 1 \mathrm{H}, \mathrm{H}-3), 2.16$ (ddd, $J=1.8,5.9,13.7 \mathrm{~Hz}, 1 \mathrm{H}, \mathrm{H}-3$ '), 2.48-2.59 (m, 1H, H-4), 4.91 (dd, $J=1.8,11.5 \mathrm{~Hz}, 1 \mathrm{H}, \mathrm{H}-2), 6.98$ (d, $J=1.8 \mathrm{~Hz}, 1 \mathrm{H}, \mathrm{H}-6), 7.10-7.40$ (m, 10H). ${ }^{13} \mathrm{C}$ NMR $(\delta \mathrm{ppm}) 20.7,32.2(\mathrm{C}-4), 40.9$ (C-3), 78.6 (C-2), 109.0 (C-5), 126.0, 126.2, 128.1, 128.6, 129.1, 129.9, 132.7, 140.9, 151.9 (C-6). IR (KBr): 3057, 1694, 1607, 1577, 30 1477, 1438, 1388, 1369, 1167, 1068, 1022, 737, $691 \mathrm{~cm}^{-1}$; MS (EI, $\left.70 \mathrm{eV}\right) \mathrm{m} / \mathrm{z}: 330$ (34), 328 (18), 226 (100), 224 (50), 207 (26), 157 (36, $\left.\mathrm{PhSe}^{+}\right), 117$ (44), $115(40), 105$ (42), $77\left(52, \mathrm{Ph}^{+}\right), 51$ (46), 44 (38).

Typical procedure for the synthesis of selenoxides (4).

To a mixture of dihydropyran 3a $(1 \mathrm{mmol}, 297 \mathrm{mg})$ in tetrahydrofuran $(10 \mathrm{ml})$, was added slowly $\mathrm{H}_{2} \mathrm{O}_{2} 35 \%$ w in water $(0.4 \mathrm{ml}, 4 \mathrm{eq}$. $)$ at 35 room temperature. After the reaction was completed, controlled by TLC (usually 2 hours), the layers were separated and the organic one dried and concentrated under vacuum. The residue was chromatographied on silicagel (eluent: MeOH/ EtOAc: 1/99) to give 200 mg (64 $\%$ ) of 2-Ethoxy-4-methyl-5-phenylseleninyl-3,4-dihydro-2H-pyran 4a.

2-Ethoxy-4-methyl-5-phenylseleninyl-3,4-dihydro-2 $H$-pyran (4a). mixture of 2 diastereomers: $53 / 47$. yield $=64 \%$; yellow oil; $R_{f}=0.31$ $(\mathrm{MeOH} / \mathrm{AcOEt}: 1 / 99) ;{ }^{1} \mathrm{H}$ NMR $\delta(\mathrm{ppm})(0.90,1.26)(\mathrm{d}, J=7.0 \mathrm{~Hz}, 3 \mathrm{H}), 1.21(\mathrm{t}, J=7.0 \mathrm{~Hz}, 3 \mathrm{H}), 1.76(\mathrm{~m}, 1 \mathrm{H}, \mathrm{H}-3), 1.94(\mathrm{~m}, 1 \mathrm{H}, \mathrm{H}-3)$, $40(2.41,2.55)(\mathrm{m}, 1 \mathrm{H}, \mathrm{H}-4), 3.55(\mathrm{~m}, 1 \mathrm{H}), 3.87(\mathrm{~m}, 1 \mathrm{H}), 5.05(\mathrm{dd}, J=2.6,5.1 \mathrm{~Hz}, 1 \mathrm{H}, \mathrm{H}-2),(6.91,7.04)(\mathrm{s}, 1 \mathrm{H}, \mathrm{H}-6), 7.48-7.55(\mathrm{~m}, 3 \mathrm{H})$, 7.70-7.74 (m, 2H). ${ }^{13} \mathrm{C}$ NMR $(\delta \mathrm{ppm})(15.1,15.2),(19.8,20.4),(24.4,26.4, \mathrm{C}-4),(35.4,35.7, \mathrm{C}-3),(64.7,64.8),(99.0,99.1, \mathrm{C}-2)$, (121.0, 122.2, C-5), (126.1, 126.6), (129.2, 129.6), (131.2, 131.4), (138.2, 140,1), (146.1, 148.6, C-6). IR (neat): 3418, 3054, 2930, 1732, 1622, 1456, 1442, 1378, 1279, 1139, 1096, 1076, 1021, 990, 816, 744, $691 \mathrm{~cm}^{-1}$; MS (EI, $\left.70 \mathrm{eV}\right) \mathrm{m} / \mathrm{z}: 314$ (44), 312 (40), 234 (20), 232 (12), $157\left(100, \mathrm{PhSe}^{+}\right), 154(60), 117(15), 78(16), 77\left(75, \mathrm{Ph}^{+}\right), 51(68), 50(38)$.

45 2-Ethoxy-3,4-dihydro-4,6-diphenyl-5-phenylseleninyl-2H-pyran (4I). yield=80\%; yellow oil; $R_{f}=0.34$ (AcOEt /cyclohexane: 80/20); ${ }^{1} \mathrm{H}$ NMR $\delta(\mathrm{ppm}) 1.16(\mathrm{t}, J=7.1 \mathrm{~Hz}, 3 \mathrm{H}), 2.07-2.26(\mathrm{~m}, 2 \mathrm{H}, \mathrm{H}-3), 3.54-3.65(\mathrm{~m}, 2 \mathrm{H}), 3.96(\mathrm{~m}, 1 \mathrm{H}), 5.14(\mathrm{dd}, J=2.4,7.1 \mathrm{~Hz}, 1 \mathrm{H}, \mathrm{H}-2)$, 7.10-7.53 (m, 13H), 7.73-7.78 (m, 2H). ${ }^{13} \mathrm{C}$ NMR $(\delta \mathrm{ppm}) 15.1,39.3(\mathrm{C}-4), 39.7(\mathrm{C}-3), 64.8,100.6(\mathrm{C}-2), 118.1(\mathrm{C}-5), 126.8,126.9$, 128.0, 128.4, 128.8, 129.0, 129.8, 130.6, 130.7, 134.1, 140.6, 141.3, 160.3 (C-6). IR (neat) 3418, 3061, 2976, 2931, 2360, 2340, 1652, $1615,1574,1494,1455,1445,1379,1275,1134,1058,938,808,741,699 \mathrm{~cm}^{-1}$. MS (ESI, positive mode): $\mathrm{m} / \mathrm{z} 453[\mathrm{M}+\mathrm{O}+\mathrm{H}]^{+}(100)$, ${ }_{50} 451(80)$.

Typical procedure for the synthesis of tetrahydofuranones (5).

To a mixture of dihydropyran 3a (1 mmol, 297mg) in dichloromethane $(10 \mathrm{ml})$, was added slowly $\mathrm{H}_{2} \mathrm{O}_{2} 35 \%$ w in water $(0.4 \mathrm{ml}, 4 \mathrm{eq}$.) at room temperature. After the reaction was completed, controlled by TLC, the layers were separated and the organic one dried and then concentrated under vacuum. The residue was chromatographied on silicagel (eluent: Ethyl Acetate/ Cyclohexane: 10/90) to give 36 mg $55(25 \%)$ of 5-Ethoxy-3-methyl-tetrahydrofuran-2-one $\mathbf{5 a}$.

5-Ethoxy-3-methyl-tetrahydrofuran-2-one (5a). yield=25\%; yellow oil; $R_{f}=0.20$ (Cyclohexane/EtOAc: 80/20), ${ }^{1} \mathrm{H}$ NMR $\delta(\mathrm{ppm}) 1.18$ $1.27(\mathrm{~m}, 6 \mathrm{H}), 1.97(\mathrm{~m}, 1 \mathrm{H}, \mathrm{H}-4), 2.35(\mathrm{~m}, 1 \mathrm{H}, \mathrm{H}-4), 2.83(\mathrm{~m}, 1 \mathrm{H}, \mathrm{H}-3), 3.58(\mathrm{~m}, 1 \mathrm{H}), 3.83(\mathrm{~m}, 1 \mathrm{H}), 5.44(\mathrm{~d}, 1 \mathrm{H}, J=5.6 \mathrm{~Hz}) ;{ }^{13} \mathrm{C} \mathrm{NMR}(\delta$ ppm) 14.95, 15.25, 32.7 (C-3), 37.3 (C-4), 64.94, 101.8 (C-5), 180.0 (C-2). IR (neat): 2978, 2936, 1778, 1738, 1446, 1378, 1349, 1213, 1169, 1112, 1040, 981, 951, $917 \mathrm{~cm}^{-1}$; MS (EI, $\left.70 \mathrm{eV}\right) \mathrm{m} / \mathrm{z}: 143$ (2), 100 (42), 99 (32), 85 (43), 72 (19), 57 (96), 43 (95), 42 (95), 41 60 (100). Anal. Calcd for $\mathrm{C}_{7} \mathrm{H}_{12} \mathrm{O}_{3}$ (144.166): C, 58.31; H, 8.39. Found: $\mathrm{C}, 57.96 ; \mathrm{H}, 8.43$.

5-Ethoxy-3-((tert-butyl)dimethylsilyl-methoxy)-tetrahydrofuran-2-one (5b). yield=42\%; yellow oil; $R_{f}=0.35$ (Cyclohexane/EtOAc: 90/10), major trans isomer: ${ }^{1} \mathrm{H}$ NMR $\delta(\mathrm{ppm}) 0.05(\mathrm{~s}, 6 \mathrm{H}), 0.87(\mathrm{~s}, 9 \mathrm{H}), 1.22(\mathrm{t}, 3 \mathrm{H}, J=7.1 \mathrm{~Hz}), 2.19$ (ddd, 1H, $\left.J=1.6,9.4,13.4 \mathrm{~Hz}, \mathrm{H}-4\right)$, 2.46 (ddd, $1 \mathrm{H}, J=5.9,8.6,13.4 \mathrm{~Hz}, \mathrm{H}-4$ '), 2.88 (m, $1 \mathrm{H}, \mathrm{H}-3), 3.59$ (dq, $J=7.1,9.5 \mathrm{~Hz}, 1 \mathrm{H}), 3.75$ (dd, $J=3.1,9.9 \mathrm{~Hz}, 1 \mathrm{H}), 3.86$ (dq, $J=7.0$, $9.5 \mathrm{~Hz}, 1 \mathrm{H}), 3.99$ (dd, $J=3.7,10.0 \mathrm{~Hz}, 1 \mathrm{H}), 5.48$ (dd, $1 \mathrm{H}, J=1.6,5.9 \mathrm{~Hz}, \mathrm{H}-5) ;{ }^{13} \mathrm{C}$ NMR $(\delta \mathrm{ppm})-5.5,15.0,18.2,25.8,31.6(\mathrm{C}-4), 41.6$ ${ }_{65}$ (C-3), 61.3, 65.1, 102.8 (C-5), 177.3 (C-2); IR (neat): 2956, 2931, 2858, 1782, 1472, 1446, 1377, 1349, 1259, 1116, 1004, 955, 901, 837, 779, $736 \mathrm{~cm}^{-1}$; MS (EI, $\left.70 \mathrm{eV}\right) \mathrm{m} / z: 230$ (2), 229 (8), 218 (20), 217 (100), 171 (70), 143 (94), 129 (16), 99 (24), 75 (84). Anal. Calcd for $\mathrm{C}_{13} \mathrm{H}_{26} \mathrm{O}_{4} \mathrm{Si}$ (274.43): C, 56.90; H, 9.55. Found: C, 57.18; H, 9.63.

Minor cis isomer: ${ }^{1} \mathrm{H}$ NMR $\delta(\mathrm{ppm}) 0.06(\mathrm{~s}, 6 \mathrm{H}), 0.89(\mathrm{~s}, 9 \mathrm{H}), 1.23(\mathrm{t}, 3 \mathrm{H}, J=7.1 \mathrm{~Hz}), 2.18(\mathrm{ddd}, 1 \mathrm{H}, J=4.6,7.5,13.5 \mathrm{~Hz}, \mathrm{H}-4), 2.51$ (ddd, $1 \mathrm{H}, J=6.0,9.7,13.5 \mathrm{~Hz}, \mathrm{H}-4), 2.78(\mathrm{~m}, 1 \mathrm{H}, \mathrm{H}-3), 3.63(\mathrm{dq}, 1 \mathrm{H}, J=7.1,9.3 \mathrm{~Hz}), 3.83-3.94(\mathrm{~m}, 3 \mathrm{H}), 5.50(\mathrm{dd}, J=4.6,6.0 \mathrm{~Hz}, 1 \mathrm{H}, \mathrm{H}-$ $\left.{ }_{70} 5\right) ;{ }^{13} \mathrm{C}$ NMR ( $\delta$ ppm) -5.4, 15.1, 18.4, 25.9, 31.6 (C-4), 43.2 (C-3), 62.0, 65.9, $103.6(\mathrm{C}-5), 176.2(\mathrm{C}-2)$. 
3-Methyl-tetrahydro-furo[2,3-b] furan-2-one (5c). yield=36\%; yellow oil; $R_{f}=0.39$ (cyclohexane/EtOAc: $\left.50 / 50\right),{ }^{1} \mathrm{H}$ NMR $\delta(\mathrm{ppm})$ $1.27(\mathrm{~d}, 3 \mathrm{H}, J=7.3 \mathrm{~Hz}), 1.82-2.08(\mathrm{~m}, 2 \mathrm{H}), 2.93(\mathrm{~m}, 1 \mathrm{H}, \mathrm{H}-4), 3.12(\mathrm{~m}, 1 \mathrm{H}, \mathrm{H}-3), 3.96-4.10(\mathrm{~m}, 2 \mathrm{H}), 6.00(\mathrm{~d}, 1 \mathrm{H}, J=4.8 \mathrm{~Hz}) ;{ }^{13} \mathrm{C} \mathrm{NMR}$ ( $\delta$ ppm) 11.7, 25.1, 38.2 (C-4), 44.2 (C-3), 68.9, 106.6 (C-5), 177.1 (C-2). IR (neat): 2981, 2898, 1777, 1716, 1454, 1379, 1359, 1295, 1251, 1176, 1109, 953, 907, 868, $736 \mathrm{~cm}^{-1}$; Anal. Calcd for $\mathrm{C}_{7} \mathrm{H}_{10} \mathrm{O}_{3}$ (142.15): C, 59.14; H, 7.09. Found: C, 59.08; H, 7.34.

5 Tetrahydro-furo[2,3-b]furan-2-one (5d). yield $=30 \%$; white solid; m. p. $48-50^{\circ} \mathrm{C} ; R_{f} \mathrm{f}=0.37$ (cyclohexane/EtOAc: $50 / 50$ ), ${ }^{1} \mathrm{H}$ NMR $\delta$ (ppm) 1.74-1.82 (m, 1H, H-4), 2.17-2.27 (m, 1H, H-4'), 2.44 (dd, J=3.4, 18.4 Hz, 1H, H-3), 2.88 (dd, J=10.2, 18.4 Hz, 1H, H-3'), 3.123.20 (m, 1H, H-8), 3.90-3.99 (m, 1H, H-5), 4.06-4.13 (m, 1H, H-5'), 6.08 (d, J=5.6 Hz, 1H, H-7); ${ }^{13} \mathrm{C}$ NMR $(\delta$ ppm) $32.3(\mathrm{C}-4), 35.0(\mathrm{C}-$ 3), 38.4 (C-8), 67.4 (C-5), 108.5 (C-7), 175.5 (C-2). IR (neat): 2986, 1770, 1636, 1455, 1418, 1360, 1300, 1252, 1186, 1109, 1004, 968, $871,834 \mathrm{~cm}^{-1}$; Anal. Calcd for $\mathrm{C}_{6} \mathrm{H}_{8} \mathrm{O}_{3}(128.12)$ : C, 56.24; H, 6.29. Found: C, 56.16; H, 6.43.

10 5-Ethoxy-3-phenyl-tetrahydrofuran-2-one (5e). yield=44\%; yellow oil; major trans isomer: $R_{f}=0.28$ cyclohexane/EtOAc: $\left.90 / 10\right)$, ${ }^{1} \mathrm{H}$ NMR $\delta(\mathrm{ppm}) 1.26(\mathrm{t}, 3 \mathrm{H}, J=7.1 \mathrm{~Hz}), 2.48(\mathrm{ddd}, 1 \mathrm{H}, J=5.5,11.2,13.3 \mathrm{~Hz}, \mathrm{H}-4), 2.63(\mathrm{dd}, 1 \mathrm{H}, J=8.8,13.3 \mathrm{~Hz}, \mathrm{H}-4), 3.64(\mathrm{~m}, 1 \mathrm{H}), 3.90$ $(\mathrm{m}, 1 \mathrm{H}), 4.05(\mathrm{dd}, J=8.8,11.2 \mathrm{~Hz}, 1 \mathrm{H}, \mathrm{H}-3), 5.58(\mathrm{~d}, 1 \mathrm{H}, J=5.5 \mathrm{~Hz}), 7.22-7.38(\mathrm{~m}, 5 \mathrm{H}) ;{ }^{13} \mathrm{C}$ NMR $(\delta \mathrm{ppm}) 15.1,38.4(\mathrm{C}-4), 44.3(\mathrm{C}-3)$, 65.2, 101.8 (C-5), 127.8, 128.1, 129.1, 136.6, 177.2 (C-2). IR (neat): 2978, 2931, 1775, 1734, 1498, 1456, 1378, 1342, 1206, 1140, 1108, 1040, 996, 935, 899, 752, 695, $642 \mathrm{~cm}^{-1}$; MS (EI, $\left.70 \mathrm{eV}\right) \mathrm{m} / \mathrm{z}: 207$ (24), 162 (66), 133 (56), 105 (100), 104 (31), 103 (30), 92 (10), 77 15 (35), 51 (22), 44 (48). Anal. Calcd for $\mathrm{C}_{12} \mathrm{H}_{14} \mathrm{O}_{3}$ (206.23): C, 69.88; H, 6.84. Found: C, 69.84; H, 6.85.

Minor cis isomer: ${ }^{1} \mathrm{H}$ NMR $\delta(\mathrm{ppm}) 1.29(\mathrm{t}, 3 \mathrm{H}, J=7.0 \mathrm{~Hz}), 2.33$ (ddd, $\left.1 \mathrm{H}, J=4.8,8.2,13.8 \mathrm{~Hz}, \mathrm{H}-4\right), 2.92(\mathrm{ddd}, 1 \mathrm{H}, J=5.9,10.3,13.8 \mathrm{~Hz}$, H-4'), $3.71(\mathrm{dt}, J=7.1,9.5 \mathrm{~Hz}, 1 \mathrm{H}), 3.84(\mathrm{dd}, J=8.2,10.3 \mathrm{~Hz}, 1 \mathrm{H}, \mathrm{H}-3), 3.98$ (dt, $J=7.1,9.5 \mathrm{~Hz}, 1 \mathrm{H}), 5.62(\mathrm{dd}, 1 \mathrm{H}, J=4.8,5.9 \mathrm{~Hz}, \mathrm{H}-5)$, 7.25-7.38 (m, 5H) ; ${ }^{13} \mathrm{C}$ NMR $(\delta$ ppm) 15.1, $37.1(\mathrm{C}-4), 46.0(\mathrm{C}-3), 66.1,102.9(\mathrm{C}-5), 127.7,128.2,129.0,137.0,176.0$ (C-2). IR (neat): $2978,2929,1773,1719,1498,1456,1378,1353,1146,1120,1043,996,927,753,698 \mathrm{~cm}^{-1}$.

${ }_{20}$ 5-Butoxy-3-phenyl-tetrahydrofuran-2-one (5f). yield $=24 \%$; yellow oil; $R_{f}=0.26$ cyclohexane/EtOAc: $\left.90 / 10\right),{ }^{1} \mathrm{H} \mathrm{NMR} \delta(\mathrm{ppm}) 0.94(\mathrm{t}$, $3 \mathrm{H}, J=7.3 \mathrm{~Hz}), 1.40$ (m, 2H), 1.60 (m, 2H), 2.49 (ddd, $1 \mathrm{H}, J=5.5,11.2,13.3 \mathrm{~Hz}, \mathrm{H}-4), 2.64$ (ddd, $1 \mathrm{H}, J=0.9,8.8,13.3 \mathrm{~Hz}, \mathrm{H}-4), 3.58$ (dt, $1 \mathrm{H}, J=6.6,9.5 \mathrm{~Hz}), 3.86$ (dt, 1H, $J=6.6,9.3 \mathrm{~Hz}), 4.05$ (ddd, $J=0.9,8.8,11.2 \mathrm{~Hz}, 1 \mathrm{H}, \mathrm{H}-3), 5.59$ (d, 1H, $J=5.5 \mathrm{~Hz}, \mathrm{H}-5), 7.25-7.40$ (m, 5H); ${ }^{13} \mathrm{C}$ NMR $(\delta \mathrm{ppm}) 13.9,19.3,31.5,38.4$ (C-4), 44.3 (C-3), 69.5, 102.0 (C-5), 127.8, 128.1, 129.0, 136.6, 177.2 (C-2), IR (neat): 3031, 2958, 2873, 1778, 1770, 1728, 1715, 1605, 1497, 1456, 1345, 1264, 1204, 1137, 1107, 1060, 998, 933, 800, 751, 696, 643 cm ${ }^{-1}$; $25 \mathrm{MS}$ (EI, $70 \mathrm{eV}) \mathrm{m} / \mathrm{z}$ : 191 (10), 190 (52), 161 (12), 134 (28), 133 (40), 105 (100), 92 (61), 78 (28), 77 (29), 57 (21). Anal. Calcd for $\mathrm{C}_{14} \mathrm{H}_{18} \mathrm{O}_{3}$ (234.29): C, 71.77; H, 7.74. Found: C, 72.10; H, 7.69.

5-tert-Butoxy-3-phenyl-tetrahydrofuran-2-one $(5 \mathrm{~g})$.

yield $=30 \%$; yellow oil; $R_{f}=0.20$ cyclohexane/EtOAc: $\left.90 / 10\right) ;{ }^{1} \mathrm{H}$ NMR $\delta(\mathrm{ppm}) 1.32(\mathrm{~s}, 9 \mathrm{H}), 2.53(\mathrm{~m}, 2 \mathrm{H}, \mathrm{H}-4), 4.08(\mathrm{t}, 1 \mathrm{H}, J=9.8 \mathrm{~Hz})$, $5.87(\mathrm{dd}, 1 \mathrm{H}, J=2.8,3.9 \mathrm{~Hz}), 7.24-7.38(\mathrm{~m}, 5 \mathrm{H}) ;{ }^{13} \mathrm{C}$ NMR $(\delta \mathrm{ppm}) 28.8,29.8,39.5(\mathrm{C}-4), 44.7(\mathrm{C}-3), 97.2(\mathrm{C}-5), 127.7,128.1,129.1$, $30136.9,177.3$ (C-2). IR (neat): 2979, 2930, 2854, 1778, 1770, 1738, 1732, 1606, 1498, 1455, 1395, 1368, 1338, 1266, 1180, 1138, 1102, 995, 933, 900, 858, 798, 750, 694, $641 \mathrm{~cm}^{-1}$; MS (EI, $\left.70 \mathrm{eV}\right) \mathrm{m} / \mathrm{z}: 191$ (2), 190 (8), 161 (12), 134 (61), 133 (25), 105 (40), 104 (18), 103 (18), 92 (22), 77 (16), 57(100). Anal. Calcd for $\mathrm{C}_{14} \mathrm{H}_{18} \mathrm{O}_{3}$ (234.284): C, 71.77; H, 7.74. Found: C, 71.74; H, 7.81.

5-Ethoxy-3-(4-methoxyphenyl)-tetrahydrofuran-2-one (5h). yield $=43 \%$; yellow oil; $R_{f}=0.18$ cyclohexane/EtOAc: $\left.90 / 10\right),{ }^{1} \mathrm{H}$ NMR $\delta$ (ppm) $1.26(\mathrm{t}, 3 \mathrm{H}, J=7.1 \mathrm{~Hz}), 2.46$ (ddd, $1 \mathrm{H}, J=5.7,11.1,13.2 \mathrm{~Hz}, \mathrm{H}-4), 2.62$ (ddd, 1H, $J=0.8,8.8,13.2 \mathrm{~Hz}, \mathrm{H}-4), 3.64$ (m, $1 \mathrm{H}), 3.80$ (s, $\left.{ }_{35} 3 \mathrm{H}\right), 3.90(\mathrm{~m}, 1 \mathrm{H}), 4.01(\mathrm{dd}, J=8.8,11.1 \mathrm{~Hz}, 1 \mathrm{H}, \mathrm{H}-3), 5.58(\mathrm{~d}, 1 \mathrm{H}, J=5.5 \mathrm{~Hz}), 6.87-6.92(\mathrm{~m}, 2 \mathrm{H}), 7.16-7.20(\mathrm{~m}, 2 \mathrm{H}) ;{ }^{13} \mathrm{C} \mathrm{NMR}(\delta \mathrm{ppm})$ 15.0, 38.4 (C-4), 43.4 (C-3), 55.4, 65.1, 101.7 (C-5), 114.4, 128.5, 129.2, 159.1, 177.5 (C-2); IR (neat): 2978, 2933, 1771, 1615, 1515, $1445,1342,1249,1206,1181,1142,1104,1035,936,905,831,804,781,760,736,689 \mathrm{~cm}^{-1}$; MS (EI, $\left.70 \mathrm{eV}\right) \mathrm{m} / \mathrm{z}: 236(13), 192$ (46), 163 (92), 147 (50), 135 (100), 119 (20), 105 (16), 91 (40), 77 (20), 66 (19). Anal. Calcd for $\mathrm{C}_{13} \mathrm{H}_{16} \mathrm{O}_{4}$ (236.26): C, 66.08; H, 6.83. Found: C, 66.28; H, 6.62.

40 1-(3-phenylfuran-2-yl) ethanone (6). yield=21\%; yellow oil; $R_{f}=0.4$ (AcOEt/cyclohexane: $\left.15 / 85\right)$; ${ }^{1} \mathrm{H}$ NMR $\delta(\mathrm{ppm}) 2.46(\mathrm{~s}, 3 \mathrm{H}), 6.66$ $(\mathrm{d}, 1 \mathrm{H}, J=1.6 \mathrm{~Hz}, \mathrm{H}-4), 7.36-7.44$ (m, 3H), 7.55 (d, $1 \mathrm{H}, J=1.6 \mathrm{~Hz}, \mathrm{H}-5), 7.61-7.64(\mathrm{~m}, 2 \mathrm{H}) ;{ }^{13} \mathrm{C}$ NMR $(\delta \mathrm{ppm}) 27.8,115.0(\mathrm{C}-4), 128.2$, 128.6, 129.3, 132.0, 133.5, 144.7 (C-5), 147.3, 188.0. IR (neat) 1676, 1565, 1503, 1474, 1448, 1394, 1356, 1284, 1241, 1162, 1130, 1096, 1065, 1019, 978, 932, 886, 761, 694, $639 \mathrm{~cm}^{-1}$; MS (EI, $\left.70 \mathrm{eV}\right) \mathrm{m} / \mathrm{z}: 186$ (92), 185 (75), 143 (4), 128 (4), 115 (100), 89 (30), 63 (18), 43 (20). Anal. Calcd for $\mathrm{C}_{12} \mathrm{H}_{10} \mathrm{O}_{2}$ (186.20): C, 77.40; H, 5.41. Found: C,77.04; H, 5.80.

${ }_{45}$ 4-formyloxy-2-methyl-4-phenylbutanoic acid (7s). yield=63\%; yellow oil; $R_{f}=0.66$ (AcOEt/Cyclohexane: $\left.100 / 0\right) ;{ }^{1} \mathrm{H} \mathrm{NMR} \delta(\mathrm{ppm})$ $1.15(\mathrm{~d}, J=6.8 \mathrm{~Hz}, 3 \mathrm{H}), 1.74-1.82(\mathrm{~m}, 1 \mathrm{H}, \mathrm{H}-3), 2.34-2.44(\mathrm{~m}, 2 \mathrm{H}), 5.85(\mathrm{dd}, J=5.3,6.4 \mathrm{~Hz}, 1 \mathrm{H}, \mathrm{H}-4), 7.16-7.28(\mathrm{~m}, 5 \mathrm{H}), 7.98(\mathrm{~s}, 1 \mathrm{H})$. ${ }^{13} \mathrm{C}$ NMR $(\delta \mathrm{ppm}) 17.1,36.3,39.7,73.8,126.7,128.6,128.8,139.4,160.4,182.3$. IR (neat): 2977, 2936, 2360, 2341, 1732, 1715, 1456, $1170,758,700 \mathrm{~cm}^{-1}$. MS (ESI, negative mode): m/z 443 ([2M-H]-, 100), $221\left([\mathrm{M}-\mathrm{H}]^{-}, 37\right)$. Calcd for $\mathrm{C}_{12} \mathrm{H}_{14} \mathrm{O}_{4}(222.23): \mathrm{C}, 64.85 ; \mathrm{H}$, 6.35 .

\section{Acknowledgements}

We gratefully acknowledge Dr. Isabelle Chataigner for her help in performing High Pressure Hetero-Diels-Alder reactions. The Conseil Régional Haute-Normandie is also acknowledged for financial support to S.R. through a doctoral fellowship.

\section{Notes and references}

551 Organic Synthesis, Topics in Current Chemistry Vol. 208, Springer, Berlin, Heidelberg (2000); b) T.G. Back, Organoselenium Chemistry: A Practical Approach, Oxford University Press, New York, NY (1999); c) A. Krief, L. Hevesi, Organoselenium Chemistry I, Springer, Berlin (1988); d) D. C. Liotta, Organoselenium Chemistry, Wiley, New York (1987); e) S. Patai, The Chemistry of Organic Selenium and Tellurium Compounds, Wiley, New York (1987), Vol 1 and 2; f) C. Paulmier, Selenium Reagents and Intermediates in Organic Synthesis, Pergamon, Oxford (1986); g) T. Wirth, Organoselenium Chemistry: Modern developments in Organic Synthesis; Springer: Berlin, (2000).

602 a) C. Paulmier, Phosphorus, Sulfur and Silicon, 2001, 172, 25 ; b) C. Miniejew, F. Outurquin and X. Pannecoucke, Tetrahedron, 2006, 62, 2657 ; c) C. Miniejew, F. Outurquin and X. Pannecoucke, Org. Biomol. Chem. 2004, 2, 1575; d) X. Franck, E. Langlois, F. Outurquin, Synthesis, $2007,719$.

3 a) H. J. Reich, J. M. Renga, J. Org. Chem., 1975, 40, 3313; b) K. B. Sharpless, R. F. Lauer, J. Am. Chem. Soc. 1972, 94,7154

4 S. Redon, A.-L. Berthe-Berkaoui, X. Pannecoucke, F. Outurquin, Tetrahedron, 2007, 63, 3707.

5 a) H. J. Reich, M. J. Kelly, R. E. Alson, R. C. Holton, Tetrahedron, 1983, 39, 949; b) K. Uneyama, K. Takano, S. Torii, Bull. Chem. Soc. Jpn, 1983,

6556,2867 ; c) S.-S. P. Chou, C.-C. Hung, Synth Commun. 2001, 31, 1097; d) S.-S. P. Chou, C.-C. Hung, Synth Commun. $2002,32,3119$. 
6 a) J. Młochowski, M. Brząszcz, M. Giurg, J. Palus, H. Wójtowicz, Eur. J. Org. Chem. 2003, 4329; b) G.-J. ten Brink, J.-M. Vis, I. W. C. E. Arends, R. A. Sheldon, J. Org. Chem. 2001, 66, 2429.

7 Such an intermediate has been observed during oxidation of cyclohexene to adipic acid, see: S.-O. Lee, R. Raja, K. D. M. Harris, J. M. Thomas, B. F. G. Johnson, G. Sankar, Angew. Chem. Int. Ed. 2003, 42, 1520.

58 A. Armstrong, H. Chung, Tetrahedron Lett. 2006, 47, 1617.

9 D. Enders, J. Vazquez, G. Raabe, Eur. J. Org. Chem. 2000, 893-901. 